Preprint typeset in JHEP style - HYPER VERSION

\title{
Generalized parton correlation functions for a spin-1/2 hadron
}

\author{
Stephan Meißner \\ Institut für Theoretische Physik II, Ruhr-Universität Bochum, \\ 44780 Bochum, Germany \\ E-mail: stephan.meissner@tp2.rub.de \\ Andreas Metz \\ Department of Physics, Temple University, \\ Philadelphia, PA 19122-6082, U.S.A. \\ E-mail: metza@temple.edu \\ Marc Schlegel \\ Theory Center, Jefferson Lab, 12000 Jefferson Avenue, \\ Newport News, VA 23606, U.S.A. \\ E-mail: schlegel@jlab.org
}

\begin{abstract}
The fully unintegrated, off-diagonal quark-quark correlator for a spin-1/2 hadron is parameterized in terms of so-called generalized parton correlation functions. Such objects, in particular, can be considered as mother distributions of generalized parton distributions on the one hand and transverse momentum dependent parton distributions on the other. Therefore, our study provides new, model-independent insights into the recently proposed nontrivial relations between generalized and transverse momentum dependent parton distributions. We find that none of these relations can be promoted to a model-independent status. As a by-product we obtain the first complete classification of generalized parton distributions beyond leading twist. The present paper is a natural extension of our previous corresponding analysis for spin-0 hadrons.
\end{abstract}

Keywords: Deep Inelastic Scattering, Hadronic Colliders, Spin and Polarization Effects, Parton Mode]. 


\section{Contents}

1. Introduction

2. Generalized parton correlation functions 3

2.1 Definition 3

2.2 Parameterization 1

2.3 Properties 6

2.4 Limits 8

3. Generalized transverse momentum dependent parton distributions 9

3.1 Definition 9

3.2 Parameterization 10

3.3 Properties 11

4. Projection of GTMDs onto TMDs and GPDs 12

4.1 TMD-limit 12

4.2 GPD-limit 14

4.3 Relations between GPDs and TMDs 18

5. Conclusions 22

A. Parameterization of Dirac bilinears 23

A.1 Parameterization of the scalar Dirac bilinear 24

A.2 Parameterization of the pseudoscalar Dirac bilinear 25

A.3 Parameterization of the vector Dirac bilinear 26

A.4 Parameterization of the axial vector Dirac bilinear 28

A.5 Parameterization of the tensor Dirac bilinear 30

B. Relations between GTMDs and GPCFs 33

G. Model calculation of GTMDs 34

\section{Introduction}

In a recent work we parameterized the fully unintegrated, off-diagonal quark-quark correlator for a spin-0 hadron in terms of so-called generalized parton correlation functions (GPCFs) [1]. The GPCFs depend on the full 4-momentum of the quark and, in addition, on the momentum transfer to the hadron. As such they contain the maximum amount of information about the partonic structure of hadrons. The purpose of the present paper is 
to extend this analysis to the more interesting but at the same time more challenging case of a spin-1/2 hadron. Related work on the (simpler) unintegrated diagonal quark-quark correlator for a spin-1/2 hadron can be found in refs. [2, 3, 团.

GPCFs are of particular interest because of their connection to the generalized parton distributions (GPDs) [5, 6, 07, 8, 9, 10, 11] and the transverse momentum dependent parton distributions (TMDs) [12, 13, 14, 15]. Both GPDs and TMDs have been intensely studied during the last 15 years. While GPDs appear in the QCD-description of hard exclusive reactions such as deep virtual Compton scattering or hard exclusive meson production, TMDs can be measured in certain semi-inclusive reactions like semi-inclusive deep inelastic scattering (SIDIS) or the Drell-Yan (DY) process. These two types of parton distributions provide a 3-dimensional picture of the nucleon - either in a mixed position-momentum representation or in pure momentum space. Moreover, they contain important information on the orbital motion of partons inside the nucleon. The important point is that both the GPDs and the TMDs appear as two different limiting cases of the GPCFs. Therefore, the GPCFs can be considered as mother distributions of GPDs and TMDs [16, 17, 10]. Note that the GPCFs also have a direct connection to the so-called Wigner distributions the quantum mechanical analogues of classical phase space distributions - of the hadronparton system [16, 17, 10].

In the present paper, as the major application of the classification of the GPCFs, we obtain new, model-independent information on the nontrivial relations between GPDs and TMDs which have been suggested in the literature 18, 19, 20, 21, 22, 23, 24, 25]. In order to study this point we exploit the connection between the GPCFs on the one hand as well as the GPDs and TMDs on the other, and explore, in particular, which GPDs and TMDs have the same mother distributions. The nontrivial relations between GPDs and TMDs attracted a lot of attention during the last years. The most prominent case, first proposed in ref. [18], is the relation between the so-called Sivers TMD [26, 27] and the GPD E. This connection provides a rather intuitive understanding of the Sivers single spin asymmetry in SIDIS which has been explored by the HERMES and the COMPASS experiments [28, 29, 30, 31]. Although in the meantime various nontrivial relations between GPDs and TMDs were established in simple models (see [24] for an overview and [25]), no model-independent relations have been obtained so far. In fact, our previous work on GPCFs showed that for spin-0 hadrons no model-independent relations between GPDs and TMDs can be established. In the present work we arrive at the same conclusion for spin- $1 / 2$ hadrons. A first account on the spin-1/2 case can be found in the conference contribution [32].

If the GPCFs are integrated upon one light-cone component of the quark momentum one arrives at the so-called generalized transverse momentum dependent parton distributions (GTMDs) which can show up in the description of hard exclusive reactions. While quark GTMDs typically appear at subleading twist - and in cases where the standard collinear factorization cannot be applied - (see, e.g., refs. 33, 34, 35]), gluon GTMDs have been extensively used to describe processes at high energies (low $x$ ) like, for instance, diffractive vector meson [36] and Higgs production at the Tevatron and the LHC [37, 38, 39] in the framework of the so-called $k_{T}$ factorization. Also an approximate method for (theo- 
retically) constraining the unpolarized gluon GTMD has been proposed 40]. In the present work we will not further elaborate on the phenomenology of GTMDs, although it is an important topic (for related work see also refs. [3, 4).

The plan of the manuscript is as follows. In the next section the parameterization of the generalized quark-quark correlator for a spin- $1 / 2$ hadron in terms of GPCFs is presented. This parameterization forms the basis for the rest of the paper. In section 3 we consider the GTMDs. The results in that section follow in a straightforward way from those in section 2. The TMD-limit and the GPD-limit for the GTMDs are investigated in section 4, providing us with the first complete counting of GPDs beyond leading twist. In particular, we also explore which GPDs and TMDs have the same mother distributions. The outcome of this analysis allows us to investigate the model-independent status of possible nontrivial relations between GPDs and TMDs. Section 5 contains the conclusions. Details of the (technically demanding) derivation of the classification for the GPCFs can be found in appendix A. The exact relations between the GPCFs and the GTMDs defined in the manuscript are given in appendix $B$, while in appendix 9 our model-independent study is supplemented by the calculation of the leading twist GTMDs in a simple diquark spectator model for the nucleon.

\section{Generalized parton correlation functions}

\subsection{Definition}

In this section we derive the structure of the generalized, fully-unintegrated quark-quark correlator for a spin- $1 / 2$ hadron which is defined as

$$
W_{\lambda \lambda^{\prime}}^{[\Gamma]}(P, k, \Delta, N ; \eta)=\frac{1}{2} \int \frac{d^{4} z}{(2 \pi)^{4}} e^{i k \cdot z}\left\langle p^{\prime}, \lambda^{\prime}\left|\bar{\psi}\left(-\frac{1}{2} z\right) \Gamma \mathcal{W}\left(-\frac{1}{2} z, \frac{1}{2} z \mid n\right) \psi\left(\frac{1}{2} z\right)\right| p, \lambda\right\rangle .
$$

The correlator $W$ depends on the helicities $\lambda$ and $\lambda^{\prime}$, the average momentum $P=\left(p+p^{\prime}\right) / 2$ of the initial and final hadron, the momentum transfer $\Delta=p^{\prime}-p$ to the hadron, and the average quark momentum $k$. (For the kinematics we also refer to figure 1.) The object $\Gamma$ is an element of the complete basis $\left\{1, \gamma_{5}, \gamma^{\mu}, \gamma^{\mu} \gamma_{5}, i \sigma^{\mu \nu}\right\}$ with $\sigma^{\mu \nu}=i\left[\gamma^{\mu}, \gamma^{\nu}\right] / 2$. The Wilson line $\mathcal{W}$ ensures the color gauge invariance of the correlator in eq. (2.1) and is running along the path ${ }^{1}$

$$
-\frac{1}{2} z \rightarrow-\frac{1}{2} z+\infty \cdot n \rightarrow \frac{1}{2} z+\infty \cdot n \rightarrow \frac{1}{2} z,
$$

with all four points connected by straight lines. It is now important to realize that the integration contour of the Wilson line not only depends on the coordinates of the initial and final points but also on the light-cone direction which is opposite to the direction of $P$ [41]. This induces a dependence on a light-cone vector $n$. In fact, instead of using $n$ a rescaled vector $\lambda n$ with some positive parameter $\lambda$ could be taken in order to specify the Wilson line. Therefore, the correlator actually only depends on the vector

$$
N=\frac{M^{2} n}{P \cdot n},
$$

\footnotetext{
${ }^{1}$ The path of the Wilson line is chosen such that appropriate Wilson lines are obtained when taking the GPD-limit and the TMD-limit (see also section 2.4).
} 


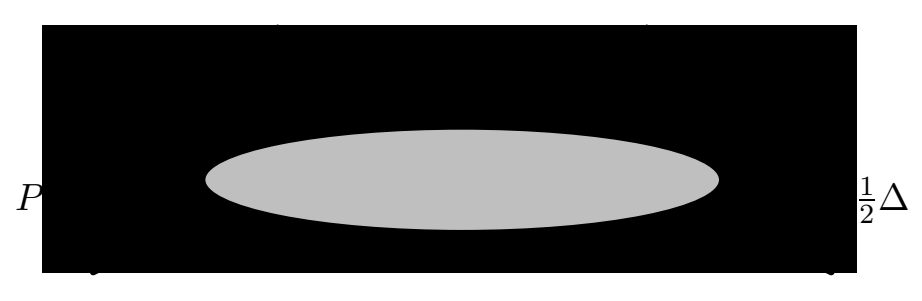

Figure 1: Kinematics for GPCFs.

which is invariant under the mentioned rescaling. For convenience in (2.3) the hadron mass $M$ is used such that $N$ has the same mass dimension as an ordinary 4-momentum. The parameter $\eta$ in (2.1) is defined through the zeroth component of $n$ according to

$$
\eta=\operatorname{sign}\left(n_{0}\right)
$$

which means that we simultaneously treat future-pointing $(\eta=+1)$ and past-pointing $(\eta=-1)$ Wilson lines. Keeping this dependence is particularly convenient once we make the projection of the correlator in (2.1) onto the correlator defining TMDs.

\subsection{Parameterization}

In order to obtain the parameterization of the correlator in (2.1) in terms of GPCFs it is necessary to analyze its behavior under parity. One finds that

$$
\begin{aligned}
& W_{\lambda \lambda^{\prime}}^{[\Gamma]}(P, k, \Delta, N ; \eta) \\
& \quad=\frac{1}{2} \int \frac{d^{4} z}{(2 \pi)^{4}} e^{i k \cdot z}\left\langle p^{\prime}, \lambda^{\prime}\left|\hat{P}^{\dagger} \hat{P} \bar{\psi}\left(-\frac{1}{2} z\right) \hat{P}^{\dagger} \hat{P} \Gamma \hat{P}^{\dagger} \hat{P} \mathcal{W}\left(-\frac{1}{2} z, \frac{1}{2} z \mid n\right) \hat{P}^{\dagger} \hat{P} \psi\left(\frac{1}{2} z\right) \hat{P}^{\dagger} \hat{P}\right| p, \lambda\right\rangle \\
& \quad=\frac{1}{2} \int \frac{d^{4} z}{(2 \pi)^{4}} e^{i k \cdot z}\left\langle\bar{p}^{\prime}, \lambda_{P}^{\prime}\left|\bar{\psi}\left(-\frac{1}{2} \bar{z}\right) \gamma_{0} \Gamma \gamma_{0} \mathcal{W}\left(-\frac{1}{2} \bar{z}, \frac{1}{2} \bar{z} \mid \bar{n}\right) \psi\left(\frac{1}{2} \bar{z}\right)\right| \bar{p}, \lambda_{P}\right\rangle \\
& \quad=\frac{1}{2} \int \frac{d^{4} z}{(2 \pi)^{4}} e^{i \bar{k} \cdot z}\left\langle\bar{p}^{\prime}, \lambda_{P}^{\prime}\left|\bar{\psi}\left(-\frac{1}{2} z\right) \gamma_{0} \Gamma \gamma_{0} \mathcal{W}\left(-\frac{1}{2} z, \frac{1}{2} z \mid \bar{n}\right) \psi\left(\frac{1}{2} z\right)\right| \bar{p}, \lambda_{P}\right\rangle \\
& =W_{\lambda_{P} \lambda_{P}^{\prime}}^{\left[\gamma_{0} \Gamma \gamma_{0}\right]}(\bar{P}, \bar{k}, \bar{\Delta}, \bar{N} ; \eta),
\end{aligned}
$$

where $\bar{P}^{\mu}=P_{\mu}=\left(P^{0},-\vec{P}\right)$ etc., while $\lambda_{P}$ and $\lambda_{P}^{\prime}$ denote the parity-reversed helicities $\lambda$ and $\lambda^{\prime}$. We now introduce the (dimensionless) matrix functions $\Gamma_{\mathrm{S}}, \Gamma_{\mathrm{P}}, \Gamma_{\mathrm{V}}^{\mu}, \Gamma_{\mathrm{A}}^{\mu}$, and $\Gamma_{\mathrm{T}}^{\mu \nu}$ through

$$
\begin{aligned}
& W_{\lambda \lambda^{\prime}}^{[1]}(P, k, \Delta, N ; \eta)=\bar{u}\left(p^{\prime}, \lambda^{\prime}\right) \Gamma_{\mathrm{S}}(P, k, \Delta, N ; \eta) u(p, \lambda) \quad \text { (scalar) }, \\
& W_{\lambda \lambda^{\prime}}^{\left[\gamma_{5}\right]}(P, k, \Delta, N ; \eta)=\bar{u}\left(p^{\prime}, \lambda^{\prime}\right) \Gamma_{\mathrm{P}}(P, k, \Delta, N ; \eta) u(p, \lambda) \quad \text { (pseudoscalar), } \\
& W_{\lambda \lambda^{\prime}}^{\left[\gamma^{\mu}\right]}(P, k, \Delta, N ; \eta)=\bar{u}\left(p^{\prime}, \lambda^{\prime}\right) \Gamma_{\mathrm{V}}^{\mu}(P, k, \Delta, N ; \eta) u(p, \lambda) \quad \text { (vector), } \\
& W_{\lambda \lambda^{\prime}}^{\left[\gamma^{\mu} \gamma_{5}\right]}(P, k, \Delta, N ; \eta)=\bar{u}\left(p^{\prime}, \lambda^{\prime}\right) \Gamma_{\mathrm{A}}^{\mu}(P, k, \Delta, N ; \eta) u(p, \lambda) \quad \text { (axial vector), } \\
& W_{\lambda \lambda^{\prime}}^{\left[i \sigma^{\mu \nu}\right]}(P, k, \Delta, N ; \eta)=\bar{u}\left(p^{\prime}, \lambda^{\prime}\right) \Gamma_{\mathrm{T}}^{\mu \nu}(P, k, \Delta, N ; \eta) u(p, \lambda) \quad \text { (tensor). }
\end{aligned}
$$


From eq. 2.5) it follows for the scalar matrix function in eq. (2.6)

$$
\begin{aligned}
& \bar{u}\left(p^{\prime}, \lambda^{\prime}\right) \Gamma_{\mathrm{S}}(P, k, \Delta, N ; \eta) u(p, \lambda) \\
& \quad=\bar{u}\left(\bar{p}^{\prime}, \lambda_{P}^{\prime}\right) \Gamma_{\mathrm{S}}(\bar{P}, \bar{k}, \bar{\Delta}, \bar{N} ; \eta) u\left(\bar{p}, \lambda_{P}\right) \\
& \quad=\bar{u}\left(p^{\prime}, \lambda^{\prime}\right) \hat{P}^{\dagger} \Gamma_{\mathrm{S}}(\bar{P}, \bar{k}, \bar{\Delta}, \bar{N} ; \eta) \hat{P} u(p, \lambda) \\
& \quad=\bar{u}\left(p^{\prime}, \lambda^{\prime}\right) \gamma_{0} \Gamma_{\mathrm{S}}(\bar{P}, \bar{k}, \bar{\Delta}, \bar{N} ; \eta) \gamma_{0} u(p, \lambda) .
\end{aligned}
$$

Analogous results hold for the other matrix functions in eqs. (2.7)-(2.10), and one finds

$$
\begin{aligned}
\Gamma_{\mathrm{S}}(P, k, \Delta, N ; \eta) & =+\gamma_{0} \Gamma_{\mathrm{S}}(\bar{P}, \bar{k}, \bar{\Delta}, \bar{N} ; \eta) \gamma_{0}, \\
\Gamma_{\mathrm{P}}(P, k, \Delta, N ; \eta) & =-\gamma_{0} \Gamma_{\mathrm{P}}(\bar{P}, \bar{k}, \bar{\Delta}, \bar{N} ; \eta) \gamma_{0}, \\
\Gamma_{\mathrm{V}}^{\mu}(P, k, \Delta, N ; \eta) & =+\gamma_{0} \Gamma_{\mathrm{V}}^{\bar{\mu}}(\bar{P}, \bar{k}, \bar{\Delta}, \bar{N} ; \eta) \gamma_{0}, \\
\Gamma_{\mathrm{A}}^{\mu}(P, k, \Delta, N ; \eta) & =-\gamma_{0} \Gamma_{\mathrm{A}}^{\bar{\mu}}(\bar{P}, \bar{k}, \bar{\Delta}, \bar{N} ; \eta) \gamma_{0}, \\
\Gamma_{\mathrm{T}}^{\mu \nu}(P, k, \Delta, N ; \eta) & =+\gamma_{0} \Gamma_{\mathrm{T}}^{\bar{\mu} \bar{\nu}}(\bar{P}, \bar{k}, \bar{\Delta}, \bar{N} ; \eta) \gamma_{0}
\end{aligned}
$$

for their behavior under parity. It turns out that the general structure of the correlator $W$ can already be obtained on the basis of the parity constraints in (2.12) $-(2.16)$. One ends up with 64 linearly independent matrix structures multiplied by scalar functions (for the derivation see appendix A],

$$
\begin{aligned}
& W_{\lambda \lambda^{\prime}}^{[1]}(P, k, \Delta, N ; \eta) \\
&= \bar{u}\left(p^{\prime}, \lambda^{\prime}\right)\left[A_{1}^{E}+\frac{i \sigma^{k \Delta}}{M^{2}} A_{2}^{E}+\frac{i \sigma^{k N}}{M^{2}} A_{3}^{E}+\frac{i \sigma^{\Delta N}}{M^{2}} A_{4}^{E}\right] u(p, \lambda) \\
& W_{\lambda \lambda^{\prime}}^{\left[\gamma_{5}\right]}(P, k, \Delta, N ; \eta) \bar{u}\left(p^{\prime}, \lambda^{\prime}\right)\left[\frac{i \varepsilon^{P k \Delta N}}{M^{4}} A_{5}^{E}+\frac{i \sigma^{P N} \gamma_{5}}{M^{2}} A_{6}^{E}+\frac{i \sigma^{k N} \gamma_{5}}{M^{2}} A_{7}^{E}+\frac{i \sigma^{\Delta N} \gamma_{5}}{M^{2}} A_{8}^{E}\right] u(p, \lambda), \\
& W_{\lambda \lambda^{\prime}}^{\left[\gamma^{\mu}\right]}(P, k, \Delta, N ; \eta) \\
&=\bar{u}\left(p^{\prime}, \lambda^{\prime}\right)\left[\frac{P^{\mu}}{M} A_{1}^{F}+\frac{k^{\mu}}{M} A_{2}^{F}+\frac{\Delta^{\mu}}{M} A_{3}^{F}+\frac{N^{\mu}}{M} A_{4}^{F}+\frac{i \sigma^{\mu k}}{M} A_{5}^{F}+\frac{i \sigma^{\mu \Delta}}{M} A_{6}^{F}+\frac{i \sigma^{\mu N}}{M} A_{7}^{F}\right. \\
& \quad+\frac{P^{\mu} i \sigma^{k \Delta}}{M^{3}} A_{8}^{F}+\frac{k^{\mu} i \sigma^{k \Delta}}{M^{3}} A_{9}^{F}+\frac{N^{\mu} i \sigma^{k \Delta}}{M^{3}} A_{10}^{F}+\frac{P^{\mu} i \sigma^{k N}}{M^{3}} A_{11}^{F}+\frac{k^{\mu} i \sigma^{k N}}{M^{3}} A_{12}^{F} \\
&\left.\quad+\frac{N^{\mu} i \sigma^{k N}}{M^{3}} A_{13}^{F}+\frac{P^{\mu} i \sigma^{\Delta N}}{M^{3}} A_{14}^{F}+\frac{\Delta^{\mu} i \sigma^{\Delta N}}{M^{3}} A_{15}^{F}+\frac{N^{\mu} i \sigma^{\Delta N}}{M^{3}} A_{16}^{F}\right] u(p, \lambda), \\
& W_{\lambda \lambda^{\prime}}^{\left[\gamma^{\mu} \gamma_{5}\right]}(P, k, \Delta, N ; \eta) \\
&= \bar{u}\left(p^{\prime}, \lambda^{\prime}\right)\left[\frac{i \varepsilon^{\mu P k \Delta}}{M^{3}} A_{1}^{G}+\frac{i \varepsilon^{\mu P k N}}{M^{3}} A_{2}^{G}+\frac{i \varepsilon^{\mu P \Delta N}}{M^{3}} A_{3}^{G}+\frac{i \varepsilon^{\mu k \Delta N}}{M^{3}} A_{4}^{G}\right. \\
&+\frac{i \sigma^{\mu P} \gamma_{5}}{M} A_{5}^{G}+\frac{i \sigma^{\mu k} \gamma_{5}}{M} A_{6}^{G}+\frac{i \sigma^{\mu N} \gamma_{5}}{M} A_{7}^{G}+\frac{k^{\mu} i \sigma^{P N} \gamma_{5}}{M^{3}} A_{8}^{G}+\frac{\Delta^{\mu} i \sigma^{P N} \gamma_{5}}{M^{3}} A_{9}^{G} \\
&+\frac{N^{\mu} i \sigma^{P N} \gamma_{5}}{M^{3}} A_{10}^{G}+\frac{k^{\mu} i \sigma^{k N} \gamma_{5}}{M^{3}} A_{11}^{G}+\frac{\Delta^{\mu} i \sigma^{k N} \gamma_{5}}{M^{3}} A_{12}^{G}+\frac{N^{\mu} i \sigma^{k N} \gamma_{5}}{M^{3}} A_{13}^{G} \\
&\left.+\frac{P^{\mu} i \sigma^{\Delta N} \gamma_{5}}{M^{3}} A_{14}^{G}+\frac{\Delta^{\mu} i \sigma^{\Delta N} \gamma_{5}}{M^{3}} A_{15}^{G}+\frac{N^{\mu} i \sigma^{\Delta N} \gamma_{5}}{M^{3}} A_{16}^{G}\right] u(p, \lambda),
\end{aligned}
$$




$$
\begin{aligned}
W_{\lambda \lambda^{\prime}}^{\left[i \sigma^{\mu \nu}\right]}(P, k, \Delta, N ; \eta) & =\left(\delta_{\rho}^{\mu} \delta_{\sigma}^{\nu}-\delta_{\rho}^{\nu} \delta_{\sigma}^{\mu}\right) \bar{u}\left(p^{\prime}, \lambda^{\prime}\right)\left[\frac{P^{\rho} k^{\sigma}}{M^{2}} A_{1}^{H}+\frac{P^{\rho} \Delta^{\sigma}}{M^{2}} A_{2}^{H}+\frac{P^{\rho} N^{\sigma}}{M^{2}} A_{3}^{H}+\frac{k^{\rho} \Delta^{\sigma}}{M^{2}} A_{4}^{H}\right. \\
& +\frac{k^{\rho} N^{\sigma}}{M^{2}} A_{5}^{H}+\frac{\Delta^{\rho} N^{\sigma}}{M^{2}} A_{6}^{H}+i \sigma^{\rho \sigma} A_{7}^{H}+\frac{P^{\rho} i \sigma^{\sigma k}}{M^{2}} A_{8}^{H}+\frac{N^{\rho} i \sigma^{\sigma k}}{M^{2}} A_{9}^{H} \\
& +\frac{P^{\rho} i \sigma^{\sigma \Delta}}{M^{2}} A_{10}^{H}+\frac{N^{\rho} i \sigma^{\sigma \Delta}}{M^{2}} A_{11}^{H}+\frac{P^{\rho} i \sigma^{\sigma N}}{M^{2}} A_{12}^{H}+\frac{k^{\rho} i \sigma^{\sigma N}}{M^{2}} A_{13}^{H}+\frac{\Delta^{\rho} i \sigma^{\sigma N}}{M^{2}} A_{14}^{H} \\
& +\frac{N^{\rho} i \sigma^{\sigma N}}{M^{2}} A_{15}^{H}+\frac{P^{\rho} k^{\sigma} i \sigma^{k \Delta}}{M^{4}} A_{16}^{H}+\frac{P^{\rho} N^{\sigma} i \sigma^{k \Delta}}{M^{4}} A_{17}^{H}+\frac{k^{\rho} N^{\sigma} i \sigma^{k \Delta}}{M^{4}} A_{18}^{H} \\
& +\frac{P^{\rho} k^{\sigma} i \sigma^{k N}}{M^{4}} A_{19}^{H}+\frac{P^{\rho} N^{\sigma} i \sigma^{k N}}{M^{4}} A_{20}^{H}+\frac{k^{\rho} N^{\sigma} i \sigma^{k N}}{M^{4}} A_{21}^{H}+\frac{P^{\rho} \Delta^{\sigma} i \sigma^{\Delta N}}{M^{4}} A_{22}^{H} \\
& \left.+\frac{P^{\rho} N^{\sigma} i \sigma^{\Delta N}}{M^{4}} A_{23}^{H}+\frac{\Delta^{\rho} N^{\sigma} i \sigma^{\Delta N}}{M^{4}} A_{24}^{H}\right] u(p, \lambda),
\end{aligned}
$$

where we used $\varepsilon^{a b c d}=\varepsilon^{\mu \nu \rho \sigma} a_{\mu} b_{\nu} c_{\rho} d_{\sigma}$ and $\sigma^{a b}=\sigma^{\mu \nu} a_{\mu} b_{\nu}$ to shorten the notation. Our treatment leading to (2.17)-(2.21) is analogous to what has already been done for a spin-0 hadron [1]. The functions $A_{i}^{E}, A_{i}^{F}, A_{i}^{G}$, and $A_{i}^{H}$ are independent and represent the GPCFs. They depend on all possible scalar products of the momenta $P, k, \Delta$, and $N$ as well as the parameter $\eta$. The various factors of $M$ are introduced in order to assign the same mass dimension to all GPCFs. Note that the parameterizations (2.17)-(2.21) are ambiguous in the sense that one can always rewrite them into other forms by means of the Gordon identities (A.6)-(A.9). However, the amount of structures as presented in eqs. (2.17) $-(2.21)$ is minimized. For further details we refer to appendix A.

\subsection{Properties}

By applying hermiticity and time reversal to the correlator in 2.1) it is possible to derive some basic properties of the GPCFs. From hermiticity it follows that

$$
\begin{aligned}
& {\left[W_{\lambda \lambda^{\prime}}^{[\Gamma]}(P, k, \Delta, N ; \eta)\right]^{*}} \\
& \quad=\frac{1}{2} \int \frac{d^{4} z}{(2 \pi)^{4}} e^{-i k \cdot z}\left\langle p^{\prime}, \lambda^{\prime}\left|\bar{\psi}\left(-\frac{1}{2} z\right) \Gamma \mathcal{W}\left(-\frac{1}{2} z, \frac{1}{2} z \mid n\right) \psi\left(\frac{1}{2} z\right)\right| p, \lambda\right\rangle^{*} \\
& \quad=\frac{1}{2} \int \frac{d^{4} z}{(2 \pi)^{4}} e^{-i k \cdot z}\left\langle p, \lambda\left|\bar{\psi}\left(\frac{1}{2} z\right) \gamma_{0} \Gamma^{\dagger} \gamma_{0} \mathcal{W}\left(\frac{1}{2} z,-\frac{1}{2} z \mid n\right) \psi\left(-\frac{1}{2} z\right)\right| p^{\prime}, \lambda^{\prime}\right\rangle \\
& \quad=\frac{1}{2} \int \frac{d^{4} z}{(2 \pi)^{4}} e^{i k \cdot z}\left\langle p, \lambda\left|\bar{\psi}\left(-\frac{1}{2} z\right) \gamma_{0} \Gamma^{\dagger} \gamma_{0} \mathcal{W}\left(-\frac{1}{2} z, \frac{1}{2} z \mid n\right) \psi\left(\frac{1}{2} z\right)\right| p^{\prime}, \lambda^{\prime}\right\rangle \\
& \quad=W_{\lambda^{\prime} \lambda}^{\left[\gamma_{0} \Gamma^{\dagger} \gamma_{0}\right]}(P, k,-\Delta, N ; \eta) .
\end{aligned}
$$

For the matrix functions in eqs. (2.6) -2.10 ) this leads to

$$
\begin{aligned}
& {\left[\Gamma_{\mathrm{S}}(P, k, \Delta, N ; \eta)\right]^{\dagger}=+\gamma_{0} \Gamma_{\mathrm{S}}(P, k,-\Delta, N ; \eta) \gamma_{0},} \\
& {\left[\Gamma_{\mathrm{P}}(P, k, \Delta, N ; \eta)\right]^{\dagger}=-\gamma_{0} \Gamma_{\mathrm{P}}(P, k,-\Delta, N ; \eta) \gamma_{0},} \\
& {\left[\Gamma_{\mathrm{V}}^{\mu}(P, k, \Delta, N ; \eta)\right]^{\dagger}=+\gamma_{0} \Gamma_{\mathrm{V}}^{\mu}(P, k,-\Delta, N ; \eta) \gamma_{0},}
\end{aligned}
$$




$$
\begin{gathered}
{\left[\Gamma_{\mathrm{A}}^{\mu}(P, k, \Delta, N ; \eta)\right]^{\dagger}=+\gamma_{0} \Gamma_{\mathrm{A}}^{\mu}(P, k,-\Delta, N ; \eta) \gamma_{0},} \\
{\left[\Gamma_{\mathrm{T}}^{\mu \nu}(P, k, \Delta, N ; \eta)\right]^{\dagger}=-\gamma_{0} \Gamma_{\mathrm{T}}^{\mu \nu}(P, k,-\Delta, N ; \eta) \gamma_{0} .}
\end{gathered}
$$

Applying the hermiticity constraints (2.23)-(2.27) to the decomposition in (2.17)-(2.21) one finds

$$
X^{*}(P, k, \Delta, N ; \eta)= \pm X(P, k,-\Delta, N ; \eta),
$$

where the plus sign holds for $X=A_{1}^{E}, A_{2}^{E}, A_{4}^{E}, A_{8}^{E}, A_{1}^{F}, A_{2}^{F}, A_{4}^{F}, A_{6}^{F}, A_{8}^{F}, A_{9}^{F}, A_{10}^{F}, A_{14}^{F}$, $A_{16}^{F}, A_{1}^{G}, A_{3}^{G}, A_{4}^{G}, A_{5}^{G}, A_{6}^{G}, A_{7}^{G}, A_{8}^{G}, A_{10}^{G}, A_{11}^{G}, A_{13}^{G}, A_{15}^{G}, A_{2}^{H}, A_{4}^{H}, A_{6}^{H}, A_{7}^{H}, A_{8}^{H}, A_{9}^{H}, A_{12}^{H}$, $A_{13}^{H}, A_{15}^{H}, A_{19}^{H}, A_{20}^{H}, A_{21}^{H}, A_{22}^{H}, A_{24}^{H}$ and the minus sign for all the other GPCFs.

In addition, time reversal leads to

$$
\begin{aligned}
& {\left[W_{\lambda \lambda^{\prime}}^{[\Gamma]}(P, k, \Delta, N ; \eta)\right]^{*}} \\
& \quad=\frac{1}{2} \int \frac{d^{4} z}{(2 \pi)^{4}} e^{-i k \cdot z}\left\langle p^{\prime}, \lambda^{\prime}\left|\bar{\psi}\left(-\frac{1}{2} z\right) \Gamma \mathcal{W}\left(-\frac{1}{2} z, \frac{1}{2} z \mid n\right) \psi\left(\frac{1}{2} z\right)\right| p, \lambda\right\rangle^{*} \\
& \quad=\frac{1}{2} \int \frac{d^{4} z}{(2 \pi)^{4}} e^{-i k \cdot z}\left\langle p^{\prime}, \lambda^{\prime}\left|\hat{T}^{\dagger} \hat{T} \bar{\psi}\left(-\frac{1}{2} z\right) \hat{T}^{\dagger} \hat{T} \Gamma \hat{T}^{\dagger} \hat{T} \mathcal{W}\left(-\frac{1}{2} z, \frac{1}{2} z \mid n\right) \hat{T}^{\dagger} \hat{T} \psi\left(\frac{1}{2} z\right) \hat{T}^{\dagger} \hat{T}\right| p, \lambda\right\rangle \\
& \quad=\frac{1}{2} \int \frac{d^{4} z}{(2 \pi)^{4}} e^{-i k \cdot z}\left\langle\bar{p}^{\prime}, \lambda_{T}^{\prime}\left|\bar{\psi}\left(\frac{1}{2} \bar{z}\right)\left(-i \gamma_{5} C\right) \Gamma^{*}\left(-i \gamma_{5} C\right) \mathcal{W}\left(\frac{1}{2} \bar{z},-\frac{1}{2} \bar{z} \mid-\bar{n}\right) \psi\left(-\frac{1}{2} \bar{z}\right)\right| \bar{p}, \lambda_{T}\right\rangle \\
& \quad=\frac{1}{2} \int \frac{d^{4} z}{(2 \pi)^{4}} e^{i \bar{k} \cdot z}\left\langle\bar{p}^{\prime}, \lambda_{T}^{\prime}\left|\bar{\psi}\left(-\frac{1}{2} z\right)\left(-i \gamma_{5} C\right) \Gamma^{*}\left(-i \gamma_{5} C\right) \mathcal{W}\left(-\frac{1}{2} z, \frac{1}{2} z \mid-\bar{n}\right) \psi\left(\frac{1}{2} z\right)\right| \bar{p}, \lambda_{T}\right\rangle \\
& \quad=W_{\lambda_{T} \lambda_{T}^{\prime}}^{\left[\left(-i \gamma_{5} C\right) \Gamma^{*}\left(-i \gamma_{5} C\right)\right]}(\bar{P}, \bar{k}, \bar{\Delta}, \bar{N} ;-\eta),
\end{aligned}
$$

where $C$ is the charge conjugation matrix, while $\lambda_{T}$ and $\lambda_{T}^{\prime}$ denote the time-reversed helicities $\lambda$ and $\lambda^{\prime}$. Analogous to eq. (2.11) one finds for the matrix functions in eqs. (2.6)(2.10)

$$
\begin{aligned}
& {\left[\Gamma_{\mathrm{S}}(P, k, \Delta, N ; \eta)\right]^{*}=\left(-i \gamma_{5} C\right) \Gamma_{\mathrm{S}}(\bar{P}, \bar{k}, \bar{\Delta}, \bar{N} ;-\eta)\left(-i \gamma_{5} C\right), } \\
& {\left[\Gamma_{\mathrm{P}}(P, k, \Delta, N ; \eta)\right]^{*}=\left(-i \gamma_{5} C\right) \Gamma_{\mathrm{P}}(\bar{P}, \bar{k}, \bar{\Delta}, \bar{N} ;-\eta)\left(-i \gamma_{5} C\right), } \\
& {\left[\Gamma_{\mathrm{V}}^{\mu}(P, k, \Delta, N ; \eta)\right]^{*}=\left(-i \gamma_{5} C\right) \Gamma_{\mathrm{V}}^{\bar{\mu}}(\bar{P}, \bar{k}, \bar{\Delta}, \bar{N} ;-\eta)\left(-i \gamma_{5} C\right), } \\
& {\left[\Gamma_{\mathrm{A}}^{\mu}(P, k, \Delta, N ; \eta)\right]^{*}=\left(-i \gamma_{5} C\right) \Gamma_{\mathrm{A}}^{\bar{\mu}}(\bar{P}, \bar{k}, \bar{\Delta}, \bar{N} ;-\eta)\left(-i \gamma_{5} C\right), } \\
& {\left[\Gamma_{\mathrm{T}}^{\mu \nu}(P, k, \Delta, N ; \eta)\right]^{*}=\left(-i \gamma_{5} C\right) \Gamma_{\mathrm{T}}^{\bar{\mu} \bar{\nu}}(\bar{P}, \bar{k}, \bar{\Delta}, \bar{N} ;-\eta)\left(-i \gamma_{5} C\right) . }
\end{aligned}
$$

The time-reversal constraints (2.30)-(2.34) provide

$$
X^{*}(P, k, \Delta, N ; \eta)=X(P, k, \Delta, N ;-\eta)
$$

for all GPCFs, relating those defined with future-pointing Wilson lines to those defined with past-pointing lines.

From these considerations it follows that in general GPCFs, unlike GPDs or TMDs, are complex-valued functions. Keeping now in mind that $\eta \in\{-1,1\}$ and using eq. 2.35) 
one finds immediately that only the imaginary part of the GPCFs depends on $\eta$. This allows one to write

$$
X(P, k, \Delta, N ; \eta)=X^{e}(P, k, \Delta, N)+i X^{o}(P, k, \Delta, N ; \eta)
$$

with

$$
X^{o}(P, k, \Delta, N ; \eta)=-X^{o}(P, k, \Delta, N ;-\eta),
$$

where we call $X^{e}$ the T-even and $X^{o}$ the T-odd part of the generic GPCF $X$. The sign reversal of $X^{o}$ in eq. (2.37) when going from future-pointing to past-pointing Wilson lines corresponds to the sign reversal discussed in ref. 42] for T-odd TMDs.

\subsection{Limits}

Now we would like to give a first account on the relation between GPCFs on the one hand and GPDs as well as TMDs on the other. To this end we consider the quark-quark correlator $F$ defining GPDs for a spin-1/2 target, which can be obtained from the correlator $W$ in eq. (2.1) by means of the projection

$$
\begin{aligned}
& F_{\lambda \lambda^{\prime}}^{[\Gamma]}(P, x, \Delta, N)=\int d k^{-} d^{2} \vec{k}_{T} W_{\lambda \lambda^{\prime}}^{[\Gamma]}(P, k, \Delta, N ; \eta) \\
& \quad=\left.\frac{1}{2} \int \frac{d z^{-}}{2 \pi} e^{i k \cdot z}\left\langle p^{\prime}, \lambda^{\prime}\left|\bar{\psi}\left(-\frac{1}{2} z\right) \Gamma \mathcal{W}\left(-\frac{1}{2} z, \frac{1}{2} z \mid n\right) \psi\left(\frac{1}{2} z\right)\right| p, \lambda\right\rangle\right|_{z^{+}=\vec{z}_{T}=0} .
\end{aligned}
$$

In this formula we use light-cone components that are specified through $a^{ \pm}=\left(a^{0} \pm a^{3}\right) / \sqrt{2}$ and $\vec{a}_{T}=\left(a^{1}, a^{2}\right)$ for a generic 4-vector $a=\left(a^{0}, a^{1}, a^{2}, a^{3}\right)$, where, in particular, we choose $k^{+}=x P^{+}$. Note that after integrating upon $k^{-}$and $\vec{k}_{T}$ the dependence on the parameter $\eta$ drops out. It is well-known that in this case we are dealing with a light-cone correlator and the two quark fields are just connected by a straight line. This means that the choice of the contour in (2.2) leads, after projection, to the appropriate Wilson line for the GPDcorrelator.

The correlator $\Phi$ defining TMDs can be extracted from $W$ by putting $\Delta=0$ and integrating out one light-cone component of the quark momentum (which we choose to be $\left.k^{-}\right)$,

$$
\begin{aligned}
& \Phi_{\lambda \lambda^{\prime}}^{[\Gamma]}\left(P, x, \vec{k}_{T}, N ; \eta\right)=\int d k^{-} W_{\lambda \lambda^{\prime}}^{[\Gamma]}(P, k, 0, N ; \eta) \\
& \quad=\left.\frac{1}{2} \int \frac{d z^{-} d^{2} \vec{z}_{T}}{(2 \pi)^{3}} e^{i k \cdot z}\left\langle P, \lambda^{\prime}\left|\bar{\psi}\left(-\frac{1}{2} z\right) \Gamma \mathcal{W}\left(-\frac{1}{2} z, \frac{1}{2} z \mid n\right) \psi\left(\frac{1}{2} z\right)\right| P, \lambda\right\rangle\right|_{z^{+}=0} .
\end{aligned}
$$

Note that for $\Delta=0$ one has $p=p^{\prime}=P$. We point out that the path specified in (2.2) also leads to a proper Wilson line after taking the TMD-limit [43, 44, 45, 42, 46, 47, 48, 49, 50]. Since $\Phi$ in eq. (2.39) is not a light-cone correlator the dependence on the parameter $\eta$ remains. The case $\eta=+1$ is appropriate for defining TMDs in processes with final state interactions of the struck quark like SIDIS, while $\eta=-1$ can be used for TMDs in DY [42]. It has been emphasized in refs. 443, 51, 52, 53] that, in general, light-like Wilson lines as used in the unintegrated correlators in (2.1) and (2.39) lead to divergences. Such divergences 
can be avoided, however, by adopting a near light-cone direction. For the purpose of the present work it is sufficient to note that our general reasoning remains valid once a near light-cone direction is used instead of $n$.

It is evident that not only the correlators $F$ and $\Phi$ appear as projections of the most general two-parton correlator $W$ as outlined above, but also the GPDs and the TMDs are projections of certain GPCFs. Therefore, GPCFs can be considered as mother distributions, which actually contain the maximum amount of information on the two-parton structure of hadrons [16, 17, 10]. Despite this fact a classification of the GPCFs as given in (2.17)-(2.21) has never been worked out.

\section{Generalized transverse momentum dependent parton distributions}

\subsection{Definition}

The projections in (2.38) and (2.39) contain the integration upon the minus-component of the quark momentum. Therefore, it is useful to consider in more detail the correlator

$$
\begin{aligned}
& W_{\lambda \lambda^{\prime}}^{[\Gamma]}\left(P, x, \vec{k}_{T}, \Delta, N ; \eta\right)=\int d k^{-} W_{\lambda \lambda^{\prime}}^{[\Gamma]}(P, k, \Delta, N ; \eta) \\
& \quad=\left.\frac{1}{2} \int \frac{d z^{-} d^{2} \vec{z}_{T}}{(2 \pi)^{3}} e^{i k \cdot z}\left\langle p^{\prime}, \lambda^{\prime}\left|\bar{\psi}\left(-\frac{1}{2} z\right) \Gamma \mathcal{W}\left(-\frac{1}{2} z, \frac{1}{2} z \mid n\right) \psi\left(\frac{1}{2} z\right)\right| p, \lambda\right\rangle\right|_{z^{+}=0} .
\end{aligned}
$$

Below the parameterization of this object is given in terms of what we call generalized transverse momentum dependent parton distributions (GTMDs). Of course, this result can now be obtained in a straightforward manner on the basis of the decomposition in eqs. (2.17)-(2.21). On the basis of the above discussion it is obvious that also the GTMDs, like the GPCFs, can be considered as mother distributions of GPDs and TMDs. It is the correlator in (3.1) which for instance can enter the description of hard exclusive meson production [35], while the corresponding correlator for gluons appears when considering

diffractive processes in lepton-hadron as well as hadron-hadron collisions [36, 37, 38, 39]. The question whether or not it appears with a Wilson line as defined in (2.2) to our knowledge has never been addressed in the literature and requires further investigation that goes beyond the scope of the present work.

For our analysis we choose an infinite momentum frame such that $P$ has a large plusmomentum and no transverse momentum. The plus-component of $\Delta$ is expressed through the commonly used variable $\xi$. To be now precise the 4 -momenta in (2.17) $-(2.21)$ are specified according to

$$
\begin{aligned}
P & =\left[P^{+}, \frac{\vec{\Delta}_{T}^{2}+4 M^{2}}{8\left(1-\xi^{2}\right) P^{+}}, \overrightarrow{0}_{T}\right], \\
k & =\left[x P^{+}, k^{-}, \vec{k}_{T}\right], \\
\Delta & =\left[-2 \xi P^{+}, \frac{\xi \vec{\Delta}_{T}^{2}+4 \xi M^{2}}{4\left(1-\xi^{2}\right) P^{+}}, \vec{\Delta}_{T}\right], \\
n & =\left[0, \pm 1, \overrightarrow{0}_{T}\right] .
\end{aligned}
$$


The vector $n$ in eq. (3.5) is of course not the most general light-cone vector. In particular, it has no transverse component and points opposite to the direction of $P$ as already mentioned earlier. However, if one wants to arrive at an appropriate definition of TMDs for SIDIS and DY, there is no freedom left for this vector because it is fixed by the external momenta of the respective processes.

\subsection{Parameterization}

Now we have all the ingredients which are needed for writing down the final result for the generalized $k_{T}$-dependent correlator (3.1) in terms of GTMDs. We start with the twist-2 case for which one gets

$$
\begin{aligned}
W_{\lambda \lambda^{\prime}}^{\left[\gamma^{+}\right]}= & \frac{1}{2 M} \bar{u}\left(p^{\prime}, \lambda^{\prime}\right)\left[F_{1,1}+\frac{i \sigma^{i+} k_{T}^{i}}{P^{+}} F_{1,2}+\frac{i \sigma^{i+} \Delta_{T}^{i}}{P^{+}} F_{1,3}\right. \\
& \left.+\frac{i \sigma^{i j} k_{T}^{i} \Delta_{T}^{j}}{M^{2}} F_{1,4}\right] u(p, \lambda), \\
W_{\lambda \lambda^{\prime}}^{\left[\gamma^{+} \gamma_{5}\right]}= & \frac{1}{2 M} \bar{u}\left(p^{\prime}, \lambda^{\prime}\right)\left[-\frac{i \varepsilon_{T}^{i j} k_{T}^{i} \Delta_{T}^{j}}{M^{2}} G_{1,1}+\frac{i \sigma^{i+} \gamma_{5} k_{T}^{i}}{P^{+}} G_{1,2}+\frac{i \sigma^{i+} \gamma_{5} \Delta_{T}^{i}}{P^{+}} G_{1,3}\right. \\
& \left.+i \sigma^{+-} \gamma_{5} G_{1,4}\right] u(p, \lambda), \\
W_{\lambda \lambda^{\prime}}^{\left[i \sigma^{j+} \gamma_{5}\right]=} & \frac{1}{2 M} \bar{u}\left(p^{\prime}, \lambda^{\prime}\right)\left[-\frac{i \varepsilon_{T}^{i j} k_{T}^{i}}{M} H_{1,1}-\frac{i \varepsilon_{T}^{i j} \Delta_{T}^{i}}{M} H_{1,2}+\frac{M i \sigma^{j+} \gamma_{5}}{P^{+}} H_{1,3}\right. \\
& +\frac{k_{T}^{j} i \sigma^{k+} \gamma_{5} k_{T}^{k}}{M P^{+}} H_{1,4}+\frac{\Delta_{T}^{j} i \sigma^{k+} \gamma_{5} k_{T}^{k}}{M P^{+}} H_{1,5}+\frac{\Delta_{T}^{j} i \sigma^{k+} \gamma_{5} \Delta_{T}^{k}}{M P^{+}} H_{1,6} \\
& \left.+\frac{k_{T}^{j} i \sigma^{+-} \gamma_{5}}{M} H_{1,7}+\frac{\Delta_{T}^{j} i \sigma^{+-} \gamma_{5}}{M} H_{1,8}\right] u(p, \lambda) .
\end{aligned}
$$

Here the definitions $\varepsilon^{0123}=1$ and $\varepsilon_{T}^{i j}=\varepsilon^{-+i j}$ are used. The 16 complex-valued twist-2 GTMDs $F_{1, i}, G_{1, i}$, and $H_{1, i}$ are given by $k^{-}$-integrals of certain linear combinations of the GPCFs in (2.19) -2.21), where the explicit relations are listed in appendix B. To shorten the notation the arguments on both sides of the eqs. (3.6)-(3.8) are omitted. All GTMDs depend on the set of variables $\left(x, \xi, \vec{k}_{T}^{2}, \vec{k}_{T} \cdot \vec{\Delta}_{T}, \vec{\Delta}_{T}^{2} ; \eta\right)$.

In the twist-3 case, characterized through a suppression by one power in $P^{+}$, we find

$$
\begin{aligned}
W_{\lambda \lambda^{\prime}}^{[1]}= & \frac{1}{2 P^{+}} \bar{u}\left(p^{\prime}, \lambda^{\prime}\right)\left[E_{2,1}+\frac{i \sigma^{i+} k_{T}^{i}}{P^{+}} E_{2,2}+\frac{i \sigma^{i+} \Delta_{T}^{i}}{P^{+}} E_{2,3}\right. \\
& \left.+\frac{i \sigma^{i j} k_{T}^{i} \Delta_{T}^{j}}{M^{2}} E_{2,4}\right] u(p, \lambda) \\
W_{\lambda \lambda^{\prime}}^{\left[\gamma_{5}\right]}= & \frac{1}{2 P^{+}} \bar{u}\left(p^{\prime}, \lambda^{\prime}\right)\left[-\frac{i \varepsilon_{T}^{i j} k_{T}^{i} \Delta_{T}^{j}}{M^{2}} E_{2,5}+\frac{i \sigma^{i+} \gamma_{5} k_{T}^{i}}{P^{+}} E_{2,6}+\frac{i \sigma^{i+} \gamma_{5} \Delta_{T}^{i}}{P^{+}} E_{2,7}\right. \\
& \left.+i \sigma^{+-} \gamma_{5} E_{2,8}\right] u(p, \lambda),
\end{aligned}
$$




$$
\begin{aligned}
W_{\lambda \lambda^{\prime}}^{\left[\gamma^{j}\right]}= & \frac{1}{2 P^{+}} \bar{u}\left(p^{\prime}, \lambda^{\prime}\right)\left[\frac{k_{T}^{j}}{M} F_{2,1}+\frac{\Delta_{T}^{j}}{M} F_{2,2}+\frac{M i \sigma^{j+}}{P^{+}} F_{2,3}\right. \\
& +\frac{k_{T}^{j} i \sigma^{k+} k_{T}^{k}}{M P^{+}} F_{2,4}+\frac{\Delta_{T}^{j} i \sigma^{k+} k_{T}^{k}}{M P^{+}} F_{2,5}+\frac{\Delta_{T}^{j} i \sigma^{k+} \Delta_{T}^{k}}{M P^{+}} F_{2,6} \\
& \left.+\frac{i \sigma^{i j} k_{T}^{i}}{M} F_{2,7}+\frac{i \sigma^{i j} \Delta_{T}^{i}}{M} F_{2,8}\right] u(p, \lambda) \\
W_{\lambda \lambda^{\prime}}^{\left[\gamma^{j} \gamma_{5}\right]}= & \frac{1}{2 P^{+}} \bar{u}\left(p^{\prime}, \lambda^{\prime}\right)\left[-\frac{i \varepsilon_{T}^{i j} k_{T}^{i}}{M} G_{2,1}-\frac{i \varepsilon_{T}^{i j} \Delta_{T}^{i}}{M} G_{2,2}+\frac{M i \sigma^{j+} \gamma_{5}}{P^{+}} G_{2,3}\right. \\
& +\frac{k_{T}^{j} i \sigma^{k+} \gamma_{5} k_{T}^{k}}{M P^{+}} G_{2,4}+\frac{\Delta_{T}^{j} i \sigma^{k+} \gamma_{5} k_{T}^{k}}{M P^{+}} G_{2,5}+\frac{\Delta_{T}^{j} i \sigma^{k+} \gamma_{5} \Delta_{T}^{k}}{M P^{+}} G_{2,6} \\
& \left.+\frac{k_{T}^{j} i \sigma^{+-} \gamma_{5}}{M} G_{2,7}+\frac{\Delta_{T}^{j} i \sigma^{+-} \gamma_{5}}{M} G_{2,8}\right] u(p, \lambda) \\
W_{\lambda \lambda^{\prime}}^{\left[i \sigma^{i j} \gamma_{5}\right]=} & -\frac{i \varepsilon_{T}^{i j}}{2 P^{+}} \bar{u}\left(p^{\prime}, \lambda^{\prime}\right)\left[H_{2,1}+\frac{i \sigma^{k+} k_{T}^{k}}{P^{+}} H_{2,2}+\frac{i \sigma^{k+} \Delta_{T}^{k}}{P^{+}} H_{2,3}\right. \\
& \left.+\frac{i \sigma^{k l} k_{T}^{k} \Delta_{T}^{l}}{M^{2}} H_{2,4}\right] u(p, \lambda) \\
W_{\lambda \lambda^{\prime}}^{\left[i \sigma^{+-} \gamma_{5}\right]}= & \frac{1}{2 P^{+}} \bar{u}\left(p^{\prime}, \lambda^{\prime}\right)\left[-\frac{i \varepsilon_{T}^{i j} k_{T}^{i} \Delta_{T}^{j}}{M^{2}} H_{2,5}+\frac{i \sigma^{i+} \gamma_{5} k_{T}^{i}}{P^{+}} H_{2,6}+\frac{i \sigma^{i+} \gamma_{5} \Delta_{T}^{i}}{P^{+}} H_{2,7}\right. \\
& \left.+i \sigma^{+-} \gamma_{5} H_{2,8}\right] u(p, \lambda) .
\end{aligned}
$$

The twist- 4 result, which is basically a copy of the twist- 2 case, reads

$$
\begin{aligned}
W_{\lambda \lambda^{\prime}}^{\left[\gamma^{-}\right]}= & \frac{M}{2\left(P^{+}\right)^{2}} \bar{u}\left(p^{\prime}, \lambda^{\prime}\right)\left[F_{3,1}+\frac{i \sigma^{i+} k_{T}^{i}}{P^{+}} F_{3,2}+\frac{i \sigma^{i+} \Delta_{T}^{i}}{P^{+}} F_{3,3}\right. \\
& \left.+\frac{i \sigma^{i j} k_{T}^{i} \Delta_{T}^{j}}{M^{2}} F_{3,4}\right] u(p, \lambda) \\
W_{\lambda \lambda^{\prime}}^{\left[\gamma^{-} \gamma_{5}\right]}= & \frac{M}{2\left(P^{+}\right)^{2}} \bar{u}\left(p^{\prime}, \lambda^{\prime}\right)\left[-\frac{i \varepsilon_{T}^{i j} k_{T}^{i} \Delta_{T}^{j}}{M^{2}} G_{3,1}+\frac{i \sigma^{i+} \gamma_{5} k_{T}^{i}}{P^{+}} G_{3,2}+\frac{i \sigma^{i+} \gamma_{5} \Delta_{T}^{i}}{P^{+}} G_{3,3}\right. \\
& \left.+i \sigma^{+-} G_{3,4}\right] u(p, \lambda), \\
W_{\lambda \lambda^{\prime}}^{\left[i \sigma^{j-} \gamma_{5}\right]}= & \frac{M}{2\left(P^{+}\right)^{2}} \bar{u}\left(p^{\prime}, \lambda^{\prime}\right)\left[-\frac{i \varepsilon_{T}^{i j} k_{T}^{i}}{M} H_{3,1}-\frac{i \varepsilon_{T}^{i j} \Delta_{T}^{i}}{M} H_{3,2}+\frac{M i \sigma^{j+} \gamma_{5}}{P^{+}} H_{3,3}\right. \\
& +\frac{k_{T}^{j} i \sigma^{k+} \gamma_{5} k_{T}^{k}}{M P^{+}} H_{3,4}+\frac{\Delta_{T}^{j} i \sigma^{k+} \gamma_{5} k_{T}^{k}}{M P^{+}} H_{3,5}+\frac{\Delta_{T}^{j} i \sigma^{k+} \gamma_{5} \Delta_{T}^{k}}{M P^{+}} H_{3,6} \\
& \left.+\frac{k_{T}^{j} i \sigma^{+-} \gamma_{5}}{M} H_{3,7}+\frac{\Delta_{T}^{j} i \sigma^{+-} \gamma_{5}}{M} H_{3,8}\right] u(p, \lambda)
\end{aligned}
$$

The twist- 4 case is of course at most of academic interest but is included for completeness.

\subsection{Properties}

Like in the case of the GPCFs we also consider the implications of hermiticity and time 
reversal on the GTMDs. Hermiticity leads to

$$
X^{*}\left(x, \xi, \vec{k}_{T}^{2}, \vec{k}_{T} \cdot \vec{\Delta}_{T}, \vec{\Delta}_{T}^{2} ; \eta\right)= \pm X\left(x,-\xi, \vec{k}_{T}^{2},-\vec{k}_{T} \cdot \vec{\Delta}_{T}, \vec{\Delta}_{T}^{2} ; \eta\right)
$$

with a plus sign for $X=E_{2,1}, E_{2,3}, E_{2,4}, E_{2,7}, F_{1,1}, F_{1,3}, F_{1,4}, F_{2,1}, F_{2,5}, F_{2,8}, F_{3,1}$, $F_{3,3}, F_{3,4}, G_{1,1}, G_{1,2}, G_{1,4}, G_{2,2}, G_{2,3}, G_{2,4}, G_{2,6}, G_{2,7}, G_{3,1}, G_{3,2}, G_{3,4}, H_{1,2}, H_{1,3}, H_{1,4}$, $H_{1,6}, H_{1,7}, H_{2,2}, H_{2,5}, H_{2,6}, H_{2,8}, H_{3,2}, H_{3,3}, H_{3,4}, H_{3,6}, H_{3,7}$ and a minus sign for all the other GTMDs. These results are a direct consequence of (2.28) and the relations between GTMDs and GPCFs (see appendix B for the explicit formulas for twist-2). On the basis of (2.35) one obtains from time reversal

$$
X^{*}\left(x, \xi, \vec{k}_{T}^{2}, \vec{k}_{T} \cdot \vec{\Delta}_{T}, \vec{\Delta}_{T}^{2} ; \eta\right)=X\left(x, \xi, \vec{k}_{T}^{2}, \vec{k}_{T} \cdot \vec{\Delta}_{T}, \vec{\Delta}_{T}^{2} ;-\eta\right)
$$

for all GTMDs $X$. This means, in particular, that we can carry over eqs. (2.36) and (2.37) to the GTMD case and write

$$
X\left(x, \xi, \vec{k}_{T}^{2}, \vec{k}_{T} \cdot \vec{\Delta}_{T}, \vec{\Delta}_{T}^{2} ; \eta\right)=X^{e}\left(x, \xi, \vec{k}_{T}^{2}, \vec{k}_{T} \cdot \vec{\Delta}_{T}, \vec{\Delta}_{T}^{2}\right)+i X^{o}\left(x, \xi, \vec{k}_{T}^{2}, \vec{k}_{T} \cdot \vec{\Delta}_{T}, \vec{\Delta}_{T}^{2} ; \eta\right)
$$

with the real valued functions $X^{e}$ and $X^{o}$ respectively representing the real and imaginary part of the GTMD $X$. Only the T-odd part $X^{o}$ depends on the sign of $\eta$ according to

$$
X^{o}\left(x, \xi, \vec{k}_{T}^{2}, \vec{k}_{T} \cdot \vec{\Delta}_{T}, \vec{\Delta}_{T}^{2} ; \eta\right)=-X^{o}\left(x, \xi, \vec{k}_{T}^{2}, \vec{k}_{T} \cdot \vec{\Delta}_{T}, \vec{\Delta}_{T}^{2} ;-\eta\right),
$$

i.e., the imaginary parts of GTMDs defined with future-pointing and past-pointing Wilson lines have a reversed sign.

In order to give an estimate we have calculated the leading twist GTMDs in the scalar diquark model of the nucleon. The results are presented in appendix Q. Our treatment is restricted to lowest order in perturbation theory. To this order all T-odd parts of the GTMDs vanish - a feature which is also well-known from spectator model calculations

of T-odd TMDs. All the results listed in eqs. (C.8)-C.23) are in accordance with the hermiticity constraint (3.18).

\section{Projection of GTMDs onto TMDs and GPDs}

In this section we consider the generalized $k_{T}$-dependent correlator in eq. (3.1) for the specific TMD-kinematics and the GPD-kinematics. This procedure provides the relations between the mother distributions (GTMDs) on the one hand and the TMDs as well as the GPDs on the other. On the basis of these results one can check whether there exists model-independent support for possible nontrivial relations between GPDs and TMDs.

\subsection{TMD-limit}

We start with the TMD-limit corresponding to a vanishing momentum transfer $\Delta=0$. In this limit exactly half of the real-valued distributions vanish because they are odd as function of $\Delta$ due to the hermiticity constraint (3.18): $E_{2,1}^{o}, E_{2,2}^{e}, E_{2,3}^{o}, E_{2,4}^{o}, E_{2,5}^{e}, E_{2,6}^{e}$, $E_{2,7}^{o}, E_{2,8}^{e}, F_{1,1}^{o}, F_{1,2}^{e}, F_{1,3}^{o}, F_{1,4}^{o}, F_{2,1}^{o}, F_{2,2}^{e}, F_{2,3}^{e}, F_{2,4}^{e}, F_{2,5}^{o}, F_{2,6}^{e}, F_{2,7}^{e}, F_{2,8}^{o}, F_{3,1}^{o}, F_{3,2}^{e}, F_{3,3}^{o}$, 
$F_{3,4}^{o}, G_{1,1}^{o}, G_{1,2}^{o}, G_{1,3}^{e}, G_{1,4}^{o}, G_{2,1}^{e}, G_{2,2}^{o}, G_{2,3}^{o}, G_{2,4}^{o}, G_{2,5}^{e}, G_{2,6}^{o}, G_{2,7}^{o}, G_{2,8}^{e}, G_{3,1}^{o}, G_{3,2}^{o}, G_{3,3}^{e}$, $G_{3,4}^{o}, H_{1,1}^{e}, H_{1,2}^{o}, H_{1,3}^{o}, H_{1,4}^{o}, H_{1,5}^{e}, H_{1,6}^{o}, H_{1,7}^{o}, H_{1,8}^{e}, H_{2,1}^{e}, H_{2,2}^{o}, H_{2,3}^{e}, H_{2,4}^{e}, H_{2,5}^{o}, H_{2,6}^{o}, H_{2,7}^{e}$, $H_{2,8}^{o}, H_{3,1}^{e}, H_{3,2}^{o}, H_{3,3}^{o}, H_{3,4}^{o}, H_{3,5}^{e}, H_{3,6}^{o}, H_{3,7}^{o}, H_{3,8}^{e}$. In addition, the distributions $E_{2,3}^{e}, E_{2,4}^{e}$, $E_{2,5}^{o}, E_{2,7}^{e}, F_{1,3}^{e}, F_{1,4}^{e}, F_{2,2}^{o}, F_{2,5}^{e}, F_{2,6}^{o}, F_{2,8}^{e}, F_{3,3}^{e}, F_{3,4}^{e}, G_{1,1}^{e}, G_{1,3}^{o}, G_{2,2}^{e}, G_{2,5}^{o}, G_{2,6}^{e}, G_{2,8}^{o}, G_{3,1}^{e}$, $G_{3,3}^{o}, H_{1,2}^{e}, H_{1,5}^{o}, H_{1,6}^{e}, H_{1,8}^{o}, H_{2,3}^{o}, H_{2,4}^{o}, H_{2,5}^{e}, H_{2,7}^{o}, H_{3,2}^{e}, H_{3,5}^{o}, H_{3,6}^{e}, H_{3,8}^{o}$ do not appear in the correlator any more, because they are multiplied by a coefficient which is linear in $\Delta$. Therefore, in the TMD-limit only the following 32 (20 T-even and 12 T-odd) distributions survive: $E_{2,1}^{e}, E_{2,2}^{o}, E_{2,6}^{o}, E_{2,8}^{o}, F_{1,1}^{e}, F_{1,2}^{o}, F_{2,1}^{e}, F_{2,3}^{o}, F_{2,4}^{o}, F_{2,7}^{o}, F_{3,1}^{e}, F_{3,2}^{o}, G_{1,2}^{e}, G_{1,4}^{e}, G_{2,1}^{o}$, $G_{2,3}^{e}, G_{2,4}^{e}, G_{2,7}^{e}, G_{3,2}^{e}, G_{3,4}^{e}, H_{1,1}^{o}, H_{1,3}^{e}, H_{1,4}^{e}, H_{1,7}^{e}, H_{2,1}^{o}, H_{2,2}^{e}, H_{2,6}^{e}, H_{2,8}^{e}, H_{3,1}^{o}, H_{3,3}^{e}, H_{3,4}^{e}$, $H_{3,7}^{e}$.

The complete list of TMDs for a spin-1/2 hadron has been given in ref. [2] (see also the review article [14). Here the spin vector

$$
S=\left[\lambda \frac{P^{+}}{M},-\lambda \frac{M}{2 P^{+}}, \vec{S}_{T}\right]
$$

of the nucleon was introduced leading to the linear combination [24]

$$
\begin{aligned}
\Phi^{[\Gamma]}\left(P, x, \vec{k}_{T}, N ; S ; \eta\right)= & \frac{1+\lambda}{2} \Phi_{++}^{[\Gamma]}\left(P, x, \vec{k}_{T}, N ; \eta\right)+\frac{1-\lambda}{2} \Phi_{--}^{[\Gamma]}\left(P, x, \vec{k}_{T}, N ; \eta\right) \\
& +\frac{S_{T}^{1}-i S_{T}^{2}}{2} \Phi_{+-}^{[\Gamma]}\left(P, x, \vec{k}_{T}, N ; \eta\right)+\frac{S_{T}^{1}+i S_{T}^{2}}{2} \Phi_{-+}^{[\Gamma]}\left(P, x, \vec{k}_{T}, N ; \eta\right) .
\end{aligned}
$$

Now using the conventions of 14] for the TMDs one finds the following explicit relations between the TMDs and the GTMDs:

$$
\begin{aligned}
f_{1}\left(x, \vec{k}_{T}^{2}\right) & =F_{1,1}^{e}\left(x, 0, \vec{k}_{T}^{2}, 0,0\right), \\
f_{1 T}^{\perp}\left(x, \vec{k}_{T}^{2} ; \eta\right) & =-F_{1,2}^{o}\left(x, 0, \vec{k}_{T}^{2}, 0,0 ; \eta\right), \\
g_{1 L}\left(x, \vec{k}_{T}^{2}\right) & =G_{1,4}^{e}\left(x, 0, \vec{k}_{T}^{2}, 0,0\right), \\
g_{1 T}\left(x, \vec{k}_{T}^{2}\right) & =G_{1,2}^{e}\left(x, 0, \vec{k}_{T}^{2}, 0,0\right), \\
h_{1}^{\perp}\left(x, \vec{k}_{T}^{2} ; \eta\right) & =-H_{1,1}^{o}\left(x, 0, \vec{k}_{T}^{2}, 0,0 ; \eta\right), \\
h_{1 L}^{\perp}\left(x, \vec{k}_{T}^{2}\right) & =H_{1,7}^{e}\left(x, 0, \vec{k}_{T}^{2}, 0,0\right), \\
h_{1 T}\left(x, \vec{k}_{T}^{2}\right) & =H_{1,3}^{e}\left(x, 0, \vec{k}_{T}^{2}, 0,0\right), \\
h_{1 T}^{\perp}\left(x, \vec{k}_{T}^{2}\right) & =H_{1,4}^{e}\left(x, 0, \vec{k}_{T}^{2}, 0,0\right), \\
e\left(x, \vec{k}_{T}^{2}\right) & =E_{2,1}^{e}\left(x, 0, \vec{k}_{T}^{2}, 0,0\right), \\
e_{L}\left(x, \vec{k}_{T}^{2} ; \eta\right) & =-E_{2,8}^{o}\left(x, 0, \vec{k}_{T}^{2}, 0,0 ; \eta\right), \\
e_{T}\left(x, \vec{k}_{T}^{2} ; \eta\right) & =-E_{2,6}^{o}\left(x, 0, \vec{k}_{T}^{2}, 0,0 ; \eta\right), \\
e_{T}^{\perp}\left(x, \vec{k}_{T}^{2} ; \eta\right) & =-E_{2,2}^{o}\left(x, 0, \vec{k}_{T}^{2}, 0,0 ; \eta\right), \\
f^{\perp}\left(x, \vec{k}_{T}^{2}\right) & =F_{2,1}^{e}\left(x, 0, \vec{k}_{T}^{2}, 0,0\right), \\
f_{L}^{\perp}\left(x, \vec{k}_{T}^{2} ; \eta\right) & =F_{2,7}^{o}\left(x, 0, \vec{k}_{T}^{2}, 0,0 ; \eta\right), \\
f_{T}^{\prime}\left(x, \vec{k}_{T}^{2} ; \eta\right) & =F_{2,3}^{o}\left(x, 0, \vec{k}_{T}^{2}, 0,0 ; \eta\right), \\
f_{T}^{\perp}\left(x, \vec{k}_{T}^{2} ; \eta\right) & =F_{2,4}^{o}\left(x, 0, \vec{k}_{T}^{2}, 0,0 ; \eta\right),
\end{aligned}
$$




$$
\begin{aligned}
g^{\perp}\left(x, \vec{k}_{T}^{2} ; \eta\right) & =-G_{2,1}^{o}\left(x, 0, \vec{k}_{T}^{2}, 0,0 ; \eta\right), \\
g_{L}^{\perp}\left(x, \vec{k}_{T}^{2}\right) & =G_{2,7}^{e}\left(x, 0, \vec{k}_{T}^{2}, 0,0\right), \\
g_{T}^{\prime}\left(x, \vec{k}_{T}^{2}\right) & =G_{2,3}^{e}\left(x, 0, \vec{k}_{T}^{2}, 0,0\right), \\
g_{T}^{\perp}\left(x, \vec{k}_{T}^{2}\right) & =G_{2,4}^{e}\left(x, 0, \vec{k}_{T}^{2}, 0,0\right), \\
h\left(x, \vec{k}_{T}^{2} ; \eta\right) & =-H_{2,1}^{o}\left(x, 0, \vec{k}_{T}^{2}, 0,0 ; \eta\right), \\
h_{L}\left(x, \vec{k}_{T}^{2}\right) & =H_{2,8}^{e}\left(x, 0, \vec{k}_{T}^{2}, 0,0\right), \\
h_{T}\left(x, \vec{k}_{T}^{2}\right) & =H_{2,6}^{e}\left(x, 0, \vec{k}_{T}^{2}, 0,0\right), \\
h_{T}^{\perp}\left(x, \vec{k}_{T}^{2}\right) & =H_{2,2}^{e}\left(x, 0, \vec{k}_{T}^{2}, 0,0\right), \\
f_{3}\left(x, \vec{k}_{T}^{2}\right) & =F_{3,1}^{e}\left(x, 0, \vec{k}_{T}^{2}, 0,0\right), \\
f_{3 T}^{\perp}\left(x, \vec{k}_{T}^{2} ; \eta\right) & =-F_{3,2}^{o}\left(x, 0, \vec{k}_{T}^{2}, 0,0 ; \eta\right), \\
g_{3 L}\left(x, \vec{k}_{T}^{2}\right) & =G_{3,4}^{e}\left(x, 0, \vec{k}_{T}^{2}, 0,0\right), \\
g_{3 T}\left(x, \vec{k}_{T}^{2}\right) & =G_{3,2}^{e}\left(x, 0, \vec{k}_{T}^{2}, 0,0\right), \\
h_{3}^{\perp}\left(x, \vec{k}_{T}^{2} ; \eta\right) & =-H_{3,1}^{o}\left(x, 0, \vec{k}_{T}^{2}, 0,0 ; \eta\right), \\
h_{3 L}^{\perp}\left(x, \vec{k}_{T}^{2}\right) & =H_{3,7}^{e}\left(x, 0, \vec{k}_{T}^{2}, 0,0\right), \\
h_{3 T}\left(x, \vec{k}_{T}^{2}\right) & =H_{3,3}^{e}\left(x, 0, \vec{k}_{T}^{2}, 0,0\right), \\
h_{3 T}^{\perp}\left(x, \vec{k}_{T}^{2}\right) & =H_{3,4}^{e}\left(x, 0, \vec{k}_{T}^{2}, 0,0\right) .
\end{aligned}
$$

These results are obtained by means of eqs. (2.39) and (3.6)-(3.17). The 12 TMDs $f_{1 T}^{\perp}$, $h_{1}^{\perp}, e_{L}, e_{T}, e_{T}^{\perp}, f_{L}^{\perp}, f_{T}, f_{T}^{\perp}, g^{\perp}, h, f_{3 T}^{\perp}, h_{3}^{\perp}$ are T-odd and are related to T-odd parts of GTMDs.

\subsection{GPD-limit}

In a second step we focus on the GPD-limit which appears when integrating upon the transverse parton momentum $\vec{k}_{T}$. As already discussed after eq. (2.38) the dependence on $\eta$ drops out in this case which implies, in particular, that all effects of T-odd parts of GTMDs disappear. In the literature only the twist-2 and the chiral-even twist-3 GPDs have been introduced [54, 55]. Therefore, we give here for the first time a complete list of GPDs for all twists. The GPDs parameterize the correlator in (2.38). One finds 8 GPDs for twist-2, 16 GPDs for twist-3, and 8 GPDs for twist-4.

To be explicit the GPDs can be defined according to

$$
\begin{aligned}
F_{\lambda \lambda^{\prime}}^{\left[\gamma^{+}\right]}= & \frac{1}{2 P^{+}} \bar{u}\left(p^{\prime}, \lambda^{\prime}\right)\left[\gamma^{+} H(x, \xi, t)+\frac{i \sigma^{+\Delta}}{2 M} E(x, \xi, t)\right] u(p, \lambda), \\
F_{\lambda \lambda^{\prime}}^{\left[\gamma^{+} \gamma_{5}\right]}= & \frac{1}{2 P^{+}} \bar{u}\left(p^{\prime}, \lambda^{\prime}\right)\left[\gamma^{+} \gamma_{5} \tilde{H}(x, \xi, t)+\frac{\Delta^{+} \gamma_{5}}{2 M} \tilde{E}(x, \xi, t)\right] u(p, \lambda), \\
F_{\lambda \lambda^{\prime}}^{\left[i \sigma^{j+} \gamma_{5}\right]}= & -\frac{i \varepsilon_{T}^{i j}}{2 P^{+}} \bar{u}\left(p^{\prime}, \lambda^{\prime}\right)\left[i \sigma^{+i} H_{T}(x, \xi, t)+\frac{\gamma^{+} \Delta_{T}^{i}-\Delta^{+} \gamma^{i}}{2 M} E_{T}(x, \xi, t)\right. \\
& \left.+\frac{P^{+} \Delta_{T}^{i}-\Delta^{+} P_{T}^{i}}{M^{2}} \tilde{H}_{T}(x, \xi, t)+\frac{\gamma^{+} P_{T}^{i}-P^{+} \gamma^{i}}{M} \tilde{E}_{T}(x, \xi, t)\right] u(p, \lambda),
\end{aligned}
$$




$$
\begin{aligned}
& F_{\lambda \lambda^{\prime}}^{[1]}=\frac{M}{2\left(P^{+}\right)^{2}} \bar{u}\left(p^{\prime}, \lambda^{\prime}\right)\left[\gamma^{+} H_{2}(x, \xi, t)+\frac{i \sigma^{+\Delta}}{2 M} E_{2}(x, \xi, t)\right] u(p, \lambda), \\
& F_{\lambda \lambda^{\prime}}^{\left[\gamma_{5}\right]}=\frac{M}{2\left(P^{+}\right)^{2}} \bar{u}\left(p^{\prime}, \lambda^{\prime}\right)\left[\gamma^{+} \gamma_{5} \tilde{H}_{2}(x, \xi, t)+\frac{P^{+} \gamma_{5}}{M} \tilde{E}_{2}(x, \xi, t)\right] u(p, \lambda), \\
& F_{\lambda \lambda^{\prime}}^{\left[\gamma^{j}\right]}=\frac{M}{2\left(P^{+}\right)^{2}} \bar{u}\left(p^{\prime}, \lambda^{\prime}\right)\left[i \sigma^{+j} H_{2 T}(x, \xi, t)+\frac{\gamma^{+} \Delta_{T}^{j}-\Delta^{+} \gamma^{j}}{2 M} E_{2 T}(x, \xi, t)\right. \\
& \left.+\frac{P^{+} \Delta_{T}^{j}-\Delta^{+} P_{T}^{j}}{M^{2}} \tilde{H}_{2 T}(x, \xi, t)+\frac{\gamma^{+} P_{T}^{j}-P^{+} \gamma^{j}}{M} \tilde{E}_{2 T}(x, \xi, t)\right] u(p, \lambda), \\
& F_{\lambda \lambda^{\prime}}^{\left[\gamma^{j} \gamma_{5}\right]}=-\frac{i \varepsilon_{T}^{i j} M}{2\left(P^{+}\right)^{2}} \bar{u}\left(p^{\prime}, \lambda^{\prime}\right)\left[i \sigma^{+i} H_{2 T}^{\prime}(x, \xi, t)+\frac{\gamma^{+} \Delta_{T}^{i}-\Delta^{+} \gamma^{i}}{2 M} E_{2 T}^{\prime}(x, \xi, t)\right. \\
& \left.+\frac{P^{+} \Delta_{T}^{i}-\Delta^{+} P_{T}^{i}}{M^{2}} \tilde{H}_{2 T}^{\prime}(x, \xi, t)+\frac{\gamma^{+} P_{T}^{i}-P^{+} \gamma^{i}}{M} \tilde{E}_{2 T}^{\prime}(x, \xi, t)\right] u(p, \lambda), \\
& F_{\lambda \lambda^{\prime}}^{\left[i \sigma^{i j} \gamma_{5}\right]}=-\frac{i \varepsilon_{T}^{i j} M}{2\left(P^{+}\right)^{2}} \bar{u}\left(p^{\prime}, \lambda^{\prime}\right)\left[\gamma^{+} H_{2}^{\prime}(x, \xi, t)+\frac{i \sigma^{+\Delta}}{2 M} E_{2}^{\prime}(x, \xi, t)\right] u(p, \lambda), \\
& F_{\lambda \lambda^{\prime}}^{\left[i \sigma^{+-} \gamma_{5}\right]}=\frac{M}{2\left(P^{+}\right)^{2}} \bar{u}\left(p^{\prime}, \lambda^{\prime}\right)\left[\gamma^{+} \gamma_{5} \tilde{H}_{2}^{\prime}(x, \xi, t)+\frac{P^{+} \gamma_{5}}{M} \tilde{E}_{2}^{\prime}(x, \xi, t)\right] u(p, \lambda), \\
& F_{\lambda \lambda^{\prime}}^{\left[\gamma^{-}\right]}=\frac{M^{2}}{2\left(P^{+}\right)^{3}} \bar{u}\left(p^{\prime}, \lambda^{\prime}\right)\left[\gamma^{+} H_{3}(x, \xi, t)+\frac{i \sigma^{+\Delta}}{2 M} E_{3}(x, \xi, t)\right] u(p, \lambda), \\
& F_{\lambda \lambda^{\prime}}^{\left[\gamma^{-} \gamma_{5}\right]}=\frac{M^{2}}{2\left(P^{+}\right)^{3}} \bar{u}\left(p^{\prime}, \lambda^{\prime}\right)\left[\gamma^{+} \gamma_{5} \tilde{H}_{3}(x, \xi, t)+\frac{\Delta^{+} \gamma_{5}}{2 M} \tilde{E}_{3}(x, \xi, t)\right] u(p, \lambda), \\
& F_{\lambda \lambda^{\prime}}^{\left[i \sigma^{j-} \gamma_{5}\right]}=-\frac{i \varepsilon_{T}^{i j} M^{2}}{2\left(P^{+}\right)^{3}} \bar{u}\left(p^{\prime}, \lambda^{\prime}\right)\left[i \sigma^{+i} H_{3 T}(x, \xi, t)+\frac{\gamma^{+} \Delta_{T}^{i}-\Delta^{+} \gamma^{i}}{2 M} E_{3 T}(x, \xi, t)\right. \\
& \left.+\frac{P^{+} \Delta_{T}^{i}-\Delta^{+} P_{T}^{i}}{M^{2}} \tilde{H}_{3 T}(x, \xi, t)+\frac{\gamma^{+} P_{T}^{i}-P^{+} \gamma^{i}}{M} \tilde{E}_{3 T}(x, \xi, t)\right] u(p, \lambda),
\end{aligned}
$$

where $t=\Delta^{2}$. The structure of the traces in (4.35)-(4.46) follows readily from eqs. (3.6)(3.17) if one keeps in mind that after integrating upon $\vec{k}_{T}$ the only transverse vector left is $\vec{\Delta}_{T}$. Altogether there exist 32 GPDs corresponding to the number of TMDs. The 16 GPDs $H, E, \tilde{H}, \tilde{E}, H_{2 T}, E_{2 T}, \tilde{H}_{2 T}, \tilde{E}_{2 T}, H_{2 T}^{\prime}, E_{2 T}^{\prime}, \tilde{H}_{2 T}^{\prime}, \tilde{E}_{2 T}^{\prime}, H_{3}, E_{3}, \tilde{H}_{3}, \tilde{E}_{3}$ are chiral-even, while the remaining ones are chiral-odd. The definition of the twist-2 GPDs corresponds follows the common definition [54]. The chiral-even twist-3 GPDs $H_{2 T}, E_{2 T}, \tilde{H}_{2 T}, \tilde{E}_{2 T}$, $H_{2 T}^{\prime}, E_{2 T}^{\prime}, \tilde{H}_{2 T}^{\prime}, \tilde{E}_{2 T}^{\prime}$ are related to the functions $G_{1}, G_{2}, G_{3}, G_{4}, \tilde{G}_{1}, \tilde{G}_{2}, \tilde{G}_{3}, \tilde{G}_{4}$ that were introduced in ref. [55.

It is now straightforward to write down the following expressions for the GPDs in terms of $k_{T}$-integrals of GTMDs:

$$
\begin{aligned}
& H(x, \xi, t)=\int d^{2} \vec{k}_{T}\left[F_{1,1}^{e}+2 \xi^{2}\left(\frac{\vec{k}_{T} \cdot \vec{\Delta}_{T}}{\vec{\Delta}_{T}^{2}} F_{1,2}^{e}+F_{1,3}^{e}\right)\right], \\
& E(x, \xi, t)=\int d^{2} \vec{k}_{T}\left[-F_{1,1}^{e}+2\left(1-\xi^{2}\right)\left(\frac{\vec{k}_{T} \cdot \vec{\Delta}_{T}}{\vec{\Delta}_{T}^{2}} F_{1,2}^{e}+F_{1,3}^{e}\right)\right], \\
& \tilde{H}(x, \xi, t)=\int d^{2} \vec{k}_{T}\left[2 \xi\left(\frac{\vec{k}_{T} \cdot \vec{\Delta}_{T}}{\vec{\Delta}_{T}^{2}} G_{1,2}^{e}+G_{1,3}^{e}\right)+G_{1,4}^{e}\right],
\end{aligned}
$$




$$
\begin{aligned}
& \tilde{E}(x, \xi, t)=\int d^{2} \vec{k}_{T}\left[\frac{2\left(1-\xi^{2}\right)}{\xi}\left(\frac{\vec{k}_{T} \cdot \vec{\Delta}_{T}}{\vec{\Delta}_{T}^{2}} G_{1,2}^{e}+G_{1,3}^{e}\right)-G_{1,4}^{e}\right], \\
& H_{T}(x, \xi, t)=\int d^{2} \vec{k}_{T}\left[H_{1,3}^{e}+\frac{\vec{\Delta}_{T}^{2}}{M^{2}}\left(\frac{\left(\vec{k}_{T} \cdot \vec{\Delta}_{T}\right)^{2}}{\left(\vec{\Delta}_{T}^{2}\right)^{2}} H_{1,4}^{e}+\frac{\vec{k}_{T} \cdot \vec{\Delta}_{T}}{\vec{\Delta}_{T}^{2}} H_{1,5}^{e}+H_{1,6}^{e}\right)\right. \\
& \left.-\frac{\xi\left(\vec{\Delta}_{T}^{2}+4 M^{2}\right)}{2\left(1-\xi^{2}\right) M^{2}}\left(\frac{\vec{k}_{T} \cdot \vec{\Delta}_{T}}{\vec{\Delta}_{T}^{2}} H_{1,7}^{e}+H_{1,8}^{e}\right)\right], \\
& E_{T}(x, \xi, t)=\int d^{2} \vec{k}_{T}\left[4\left(\frac{2\left(\vec{k}_{T} \cdot \vec{\Delta}_{T}\right)^{2}-\vec{k}_{T}^{2} \vec{\Delta}_{T}^{2}}{\left(\vec{\Delta}_{T}^{2}\right)^{2}} H_{1,4}^{e}+\frac{\vec{k}_{T} \cdot \vec{\Delta}_{T}}{\vec{\Delta}_{T}^{2}} H_{1,5}^{e}+H_{1,6}^{e}\right)\right] \text {, } \\
& \tilde{H}_{T}(x, \xi, t)=\int d^{2} \vec{k}_{T}\left[\left(\frac{\vec{k}_{T} \cdot \vec{\Delta}_{T}}{\vec{\Delta}_{T}^{2}} H_{1,1}^{e}+H_{1,2}^{e}\right)\right. \\
& -2\left(1-\xi^{2}\right)\left(\frac{2\left(\vec{k}_{T} \cdot \vec{\Delta}_{T}\right)^{2}-\vec{k}_{T}^{2} \vec{\Delta}_{T}^{2}}{\left(\vec{\Delta}_{T}^{2}\right)^{2}} H_{1,4}^{e}+\frac{\vec{k}_{T} \cdot \vec{\Delta}_{T}}{\vec{\Delta}_{T}^{2}} H_{1,5}^{e}+H_{1,6}^{e}\right) \\
& \left.+\xi\left(\frac{\vec{k}_{T} \cdot \vec{\Delta}_{T}}{\vec{\Delta}_{T}^{2}} H_{1,7}^{e}+H_{1,8}^{e}\right)\right], \\
& \tilde{E}_{T}(x, \xi, t)=\int d^{2} \vec{k}_{T}\left[4 \xi\left(\frac{2\left(\vec{k}_{T} \cdot \vec{\Delta}_{T}\right)^{2}-\vec{k}_{T}^{2} \vec{\Delta}_{T}^{2}}{\left(\vec{\Delta}_{T}^{2}\right)^{2}} H_{1,4}^{e}+\frac{\vec{k}_{T} \cdot \vec{\Delta}_{T}}{\vec{\Delta}_{T}^{2}} H_{1,5}^{e}+H_{1,6}^{e}\right)\right. \\
& \left.+2\left(\frac{\vec{k}_{T} \cdot \vec{\Delta}_{T}}{\vec{\Delta}_{T}^{2}} H_{1,7}^{e}+H_{1,8}^{e}\right)\right], \\
& H_{2}(x, \xi, t)=\int d^{2} \vec{k}_{T}\left[E_{2,1}^{e}+2 \xi^{2}\left(\frac{\vec{k}_{T} \cdot \vec{\Delta}_{T}}{\vec{\Delta}_{T}^{2}} E_{2,2}^{e}+E_{2,3}^{e}\right)\right] \text {, } \\
& E_{2}(x, \xi, t)=\int d^{2} \vec{k}_{T}\left[-E_{2,1}^{e}+2\left(1-\xi^{2}\right)\left(\frac{\vec{k}_{T} \cdot \vec{\Delta}_{T}}{\vec{\Delta}_{T}^{2}} E_{2,2}^{e}+E_{2,3}^{e}\right)\right], \\
& \tilde{H}_{2}(x, \xi, t)=\int d^{2} \vec{k}_{T}\left[2 \xi\left(\frac{\vec{k}_{T} \cdot \vec{\Delta}_{T}}{\vec{\Delta}_{T}^{2}} E_{2,6}^{e}+E_{2,7}^{e}\right)+E_{2,8}^{e}\right] \text {, } \\
& \tilde{E}_{2}(x, \xi, t)=\int d^{2} \vec{k}_{T}\left[-2\left(1-\xi^{2}\right)\left(\frac{\vec{k}_{T} \cdot \vec{\Delta}_{T}}{\vec{\Delta}_{T}^{2}} E_{2,6}^{e}+E_{2,7}^{e}\right)+\xi E_{2,8}^{e}\right] \text {, } \\
& H_{2}^{\prime}(x, \xi, t)=\int d^{2} \vec{k}_{T}\left[H_{2,1}^{e}+2 \xi^{2}\left(\frac{\vec{k}_{T} \cdot \vec{\Delta}_{T}}{\vec{\Delta}_{T}^{2}} H_{2,2}^{e}+H_{2,3}^{e}\right)\right] \text {, } \\
& E_{2}^{\prime}(x, \xi, t)=\int d^{2} \vec{k}_{T}\left[-H_{2,1}^{e}+2\left(1-\xi^{2}\right)\left(\frac{\vec{k}_{T} \cdot \vec{\Delta}_{T}}{\vec{\Delta}_{T}^{2}} H_{2,2}^{e}+H_{2,3}^{e}\right)\right] \text {, } \\
& \tilde{H}_{2}^{\prime}(x, \xi, t)=\int d^{2} \vec{k}_{T}\left[2 \xi\left(\frac{\vec{k}_{T} \cdot \vec{\Delta}_{T}}{\vec{\Delta}_{T}^{2}} H_{2,6}^{e}+H_{2,7}^{e}\right)+H_{2,8}^{e}\right] \text {, } \\
& \tilde{E}_{2}^{\prime}(x, \xi, t)=\int d^{2} \vec{k}_{T}\left[-2\left(1-\xi^{2}\right)\left(\frac{\vec{k}_{T} \cdot \vec{\Delta}_{T}}{\vec{\Delta}_{T}^{2}} H_{2,6}^{e}+H_{2,7}^{e}\right)+\xi H_{2,8}^{e}\right] \text {, }
\end{aligned}
$$




$$
\begin{aligned}
& H_{2 T}(x, \xi, t)=\int d^{2} \vec{k}_{T}\left[-F_{2,3}^{e}+\frac{\left(\vec{k}_{T} \cdot \vec{\Delta}_{T}\right)^{2}-\vec{k}_{T}^{2} \vec{\Delta}_{T}^{2}}{M^{2} \vec{\Delta}_{T}^{2}} F_{2,4}^{e}\right. \\
& \left.+\frac{\xi\left(\vec{\Delta}_{T}^{2}+4 M^{2}\right)}{2\left(1-\xi^{2}\right) M^{2}}\left(\frac{\vec{k}_{T} \cdot \vec{\Delta}_{T}}{\vec{\Delta}_{T}^{2}} F_{2,7}^{e}+F_{2,8}^{e}\right)\right], \\
& E_{2 T}(x, \xi, t)=\int d^{2} \vec{k}_{T}\left[4\left(\frac{2\left(\vec{k}_{T} \cdot \vec{\Delta}_{T}\right)^{2}-\vec{k}_{T}^{2} \vec{\Delta}_{T}^{2}}{\left(\vec{\Delta}_{T}^{2}\right)^{2}} F_{2,4}^{e}+\frac{\vec{k}_{T} \cdot \vec{\Delta}_{T}}{\vec{\Delta}_{T}^{2}} F_{2,5}^{e}+F_{2,6}^{e}\right)\right] \text {, } \\
& \tilde{H}_{2 T}(x, \xi, t)=\int d^{2} \vec{k}_{T}\left[\left(\frac{\vec{k}_{T} \cdot \vec{\Delta}_{T}}{\vec{\Delta}_{T}^{2}} F_{2,1}^{e}+F_{2,2}^{e}\right)\right. \\
& -2\left(1-\xi^{2}\right)\left(\frac{2\left(\vec{k}_{T} \cdot \vec{\Delta}_{T}\right)^{2}-\vec{k}_{T}^{2} \vec{\Delta}_{T}^{2}}{\left(\vec{\Delta}_{T}^{2}\right)^{2}} F_{2,4}^{e}+\frac{\vec{k}_{T} \cdot \vec{\Delta}_{T}}{\vec{\Delta}_{T}^{2}} F_{2,5}^{e}+F_{2,6}^{e}\right) \\
& \left.-\xi\left(\frac{\vec{k}_{T} \cdot \vec{\Delta}_{T}}{\vec{\Delta}_{T}^{2}} F_{2,7}^{e}+F_{2,8}^{e}\right)\right], \\
& \tilde{E}_{2 T}(x, \xi, t)=\int d^{2} \vec{k}_{T}\left[4 \xi\left(\frac{2\left(\vec{k}_{T} \cdot \vec{\Delta}_{T}\right)^{2}-\vec{k}_{T}^{2} \vec{\Delta}_{T}^{2}}{\left(\vec{\Delta}_{T}^{2}\right)^{2}} F_{2,4}^{e}+\frac{\vec{k}_{T} \cdot \vec{\Delta}_{T}}{\vec{\Delta}_{T}^{2}} F_{2,5}^{e}+F_{2,6}^{e}\right)\right. \\
& \left.-2\left(\frac{\vec{k}_{T} \cdot \vec{\Delta}_{T}}{\vec{\Delta}_{T}^{2}} F_{2,7}^{e}+F_{2,8}^{e}\right)\right] \text {, } \\
& H_{2 T}^{\prime}(x, \xi, t)=\int d^{2} \vec{k}_{T}\left[G_{2,3}^{e}+\frac{\vec{\Delta}_{T}^{2}}{M^{2}}\left(\frac{\left(\vec{k}_{T} \cdot \vec{\Delta}_{T}\right)^{2}}{\left(\vec{\Delta}_{T}^{2}\right)^{2}} G_{2,4}^{e}+\frac{\vec{k}_{T} \cdot \vec{\Delta}_{T}}{\vec{\Delta}_{T}^{2}} G_{2,5}^{e}+G_{2,6}^{e}\right)\right. \\
& \left.-\frac{\xi\left(\vec{\Delta}_{T}^{2}+4 M^{2}\right)}{2\left(1-\xi^{2}\right) M^{2}}\left(\frac{\vec{k}_{T} \cdot \vec{\Delta}_{T}}{\vec{\Delta}_{T}^{2}} G_{2,7}^{e}+G_{2,8}^{e}\right)\right] \\
& E_{2 T}^{\prime}(x, \xi, t)=\int d^{2} \vec{k}_{T}\left[4\left(\frac{2\left(\vec{k}_{T} \cdot \vec{\Delta}_{T}\right)^{2}-\vec{k}_{T}^{2} \vec{\Delta}_{T}^{2}}{\left(\vec{\Delta}_{T}^{2}\right)^{2}} G_{2,4}^{e}+\frac{\vec{k}_{T} \cdot \vec{\Delta}_{T}}{\vec{\Delta}_{T}^{2}} G_{2,5}^{e}+G_{2,6}^{e}\right)\right], \\
& \tilde{H}_{2 T}^{\prime}(x, \xi, t)=\int d^{2} \vec{k}_{T}\left[\left(\frac{\vec{k}_{T} \cdot \vec{\Delta}_{T}}{\vec{\Delta}_{T}^{2}} G_{2,1}^{e}+G_{2,2}^{e}\right)\right. \\
& -2\left(1-\xi^{2}\right)\left(\frac{2\left(\vec{k}_{T} \cdot \vec{\Delta}_{T}\right)^{2}-\vec{k}_{T}^{2} \vec{\Delta}_{T}^{2}}{\left(\vec{\Delta}_{T}^{2}\right)^{2}} G_{2,4}^{e}+\frac{\vec{k}_{T} \cdot \vec{\Delta}_{T}}{\vec{\Delta}_{T}^{2}} G_{2,5}^{e}+G_{2,6}^{e}\right) \\
& \left.+\xi\left(\frac{\vec{k}_{T} \cdot \vec{\Delta}_{T}}{\vec{\Delta}_{T}^{2}} G_{2,7}^{e}+G_{2,8}^{e}\right)\right], \\
& \tilde{E}_{2 T}^{\prime}(x, \xi, t)=\int d^{2} \vec{k}_{T}\left[4 \xi\left(\frac{2\left(\vec{k}_{T} \cdot \vec{\Delta}_{T}\right)^{2}-\vec{k}_{T}^{2} \vec{\Delta}_{T}^{2}}{\left(\vec{\Delta}_{T}^{2}\right)^{2}} G_{2,4}^{e}+\frac{\vec{k}_{T} \cdot \vec{\Delta}_{T}}{\vec{\Delta}_{T}^{2}} G_{2,5}^{e}+G_{2,6}^{e}\right)\right. \\
& \left.+2\left(\frac{\vec{k}_{T} \cdot \vec{\Delta}_{T}}{\vec{\Delta}_{T}^{2}} G_{2,7}^{e}+G_{2,8}^{e}\right)\right], \\
& H_{3}(x, \xi, t)=\int d^{2} \vec{k}_{T}\left[F_{3,1}^{e}+2 \xi^{2}\left(\frac{\vec{k}_{T} \cdot \vec{\Delta}_{T}}{\vec{\Delta}_{T}^{2}} F_{3,2}^{e}+F_{3,3}^{e}\right)\right], \\
& E_{3}(x, \xi, t)=\int d^{2} \vec{k}_{T}\left[-F_{3,1}^{e}+2\left(1-\xi^{2}\right)\left(\frac{\vec{k}_{T} \cdot \vec{\Delta}_{T}}{\vec{\Delta}_{T}^{2}} F_{3,2}^{e}+F_{3,3}^{e}\right)\right],
\end{aligned}
$$




$$
\begin{aligned}
& \tilde{H}_{3}(x, \xi, t)=\int d^{2} \vec{k}_{T}\left[2 \xi\left(\frac{\vec{k}_{T} \cdot \vec{\Delta}_{T}}{\vec{\Delta}_{T}^{2}} G_{3,2}^{e}+G_{3,3}^{e}\right)+G_{3,4}^{e}\right], \\
& \tilde{E}_{3}(x, \xi, t)=\int d^{2} \vec{k}_{T}\left[\frac{2\left(1-\xi^{2}\right)}{\xi}\left(\frac{\vec{k}_{T} \cdot \vec{\Delta}_{T}}{\vec{\Delta}_{T}^{2}} G_{3,2}^{e}+G_{3,3}^{e}\right)-G_{3,4}^{e}\right] \text {, } \\
& H_{3 T}(x, \xi, t)=\int d^{2} \vec{k}_{T}\left[H_{3,3}^{e}+\frac{\vec{\Delta}_{T}^{2}}{M^{2}}\left(\frac{\left(\vec{k}_{T} \cdot \vec{\Delta}_{T}\right)^{2}}{\left(\vec{\Delta}_{T}^{2}\right)^{2}} H_{3,4}^{e}+\frac{\vec{k}_{T} \cdot \vec{\Delta}_{T}}{\vec{\Delta}_{T}^{2}} H_{3,5}^{e}+H_{3,6}^{e}\right)\right. \\
& \left.-\frac{\xi\left(\vec{\Delta}_{T}^{2}+4 M^{2}\right)}{2\left(1-\xi^{2}\right) M^{2}}\left(\frac{\vec{k}_{T} \cdot \vec{\Delta}_{T}}{\vec{\Delta}_{T}^{2}} H_{3,7}^{e}+H_{3,8}^{e}\right)\right], \\
& E_{3 T}(x, \xi, t)=\int d^{2} \vec{k}_{T}\left[4\left(\frac{2\left(\vec{k}_{T} \cdot \vec{\Delta}_{T}\right)^{2}-\vec{k}_{T}^{2} \vec{\Delta}_{T}^{2}}{\left(\vec{\Delta}_{T}^{2}\right)^{2}} H_{3,4}^{e}+\frac{\vec{k}_{T} \cdot \vec{\Delta}_{T}}{\vec{\Delta}_{T}^{2}} H_{3,5}^{e}+H_{3,6}^{e}\right)\right] \text {, } \\
& \tilde{H}_{3 T}(x, \xi, t)=\int d^{2} \vec{k}_{T}\left[\left(\frac{\vec{k}_{T} \cdot \vec{\Delta}_{T}}{\vec{\Delta}_{T}^{2}} H_{3,1}^{e}+H_{3,2}^{e}\right)\right. \\
& -2\left(1-\xi^{2}\right)\left(\frac{2\left(\vec{k}_{T} \cdot \vec{\Delta}_{T}\right)^{2}-\vec{k}_{T}^{2} \vec{\Delta}_{T}^{2}}{\left(\vec{\Delta}_{T}^{2}\right)^{2}} H_{3,4}^{e}+\frac{\vec{k}_{T} \cdot \vec{\Delta}_{T}}{\vec{\Delta}_{T}^{2}} H_{3,5}^{e}+H_{3,6}^{e}\right) \\
& \left.+\xi\left(\frac{\vec{k}_{T} \cdot \vec{\Delta}_{T}}{\vec{\Delta}_{T}^{2}} H_{3,7}^{e}+H_{3,8}^{e}\right)\right] \\
& \tilde{E}_{3 T}(x, \xi, t)=\int d^{2} \vec{k}_{T}\left[4 \xi\left(\frac{2\left(\vec{k}_{T} \cdot \vec{\Delta}_{T}\right)^{2}-\vec{k}_{T}^{2} \vec{\Delta}_{T}^{2}}{\left(\vec{\Delta}_{T}^{2}\right)^{2}} H_{3,4}^{e}+\frac{\vec{k}_{T} \cdot \vec{\Delta}_{T}}{\vec{\Delta}_{T}^{2}} H_{3,5}^{e}+H_{3,6}^{e}\right)\right. \\
& \left.+2\left(\frac{\vec{k}_{T} \cdot \vec{\Delta}_{T}}{\vec{\Delta}_{T}^{2}} H_{3,7}^{e}+H_{3,8}^{e}\right)\right] .
\end{aligned}
$$

The hermiticity constraint (3.18) for the GTMDs, in combination with the relations (4.47)(4.78), determines the symmetry behavior of the GPDs under the transformation $\xi \rightarrow-\xi$. One finds that the 10 GPDs $\tilde{E}_{T}, \tilde{H}_{2}, H_{2}^{\prime}, E_{2}^{\prime}, \tilde{E}_{2}^{\prime}, H_{2 T}, E_{2 T}, \tilde{H}_{2 T}, \tilde{E}_{2 T}^{\prime}, \tilde{E}_{3 T}$ are odd functions in $\xi$, while all the other GPDs are even in $\xi$. This implies that the limit $\xi \rightarrow 0$ can be performed in eqs. (4.50) and (4.74) without encountering a singularity as the GPDs $\tilde{E}$ and $\tilde{E}_{3}$ are even functions in $\xi$. In addition, note that there appears no problem when performing the limit $\vec{\Delta}_{T} \rightarrow 0$ in eqs. (4.47)-(4.78) because of

$$
\begin{gathered}
\int d^{2} \vec{k}_{T} k_{T}^{i} X\left(x, \xi, \vec{k}_{T}^{2}, \vec{k}_{T} \cdot \vec{\Delta}_{T}, \vec{\Delta}_{T}^{2} ; \eta\right) \propto \Delta_{T}^{i}, \\
\int d^{2} \vec{k}_{T}\left(2 k_{T}^{i} k_{T}^{j}-\delta_{T}^{i j} \vec{k}_{T}^{2}\right) X\left(x, \xi, \vec{k}_{T}^{2}, \vec{k}_{T} \cdot \vec{\Delta}_{T}, \vec{\Delta}_{T}^{2} ; \eta\right) \propto\left(2 \Delta_{T}^{i} \Delta_{T}^{j}-\delta_{T}^{i j} \vec{\Delta}_{T}^{2}\right),
\end{gathered}
$$

which holds for any GTMD $X$.

\subsection{Relations between GPDs and TMDs}

Having established the precise connection of the GPDs and TMDs with their respective mother distributions we are now in a position to search for possible model-independent relations between GPDs and TMDs. From (4.3) and (4.47) it is obvious that the GPD $H$ and the TMD $f_{1}$ can be related since both functions are projections of the GTMD $F_{1,1}^{e}$. 
With an analogous reasoning two additional relations can be obtained for twist-2, three for twist-3, and three for twist-4 leading altogether to

$$
\begin{aligned}
H(x, 0,0) & =\int d^{2} \vec{k}_{T} F_{1,1}^{e}\left(x, 0, \vec{k}_{T}^{2}, 0,0\right)=\int d^{2} \vec{k}_{T} f_{1}\left(x, \vec{k}_{T}^{2}\right), \\
\tilde{H}(x, 0,0) & =\int d^{2} \vec{k}_{T} G_{1,4}^{e}\left(x, 0, \vec{k}_{T}^{2}, 0,0\right)=\int d^{2} \vec{k}_{T} g_{1 L}\left(x, \vec{k}_{T}^{2}\right), \\
H_{T}(x, 0,0) & =\int d^{2} \vec{k}_{T}\left[H_{1,3}^{e}\left(x, 0, \vec{k}_{T}^{2}, 0,0\right)+\frac{\vec{k}_{T}^{2}}{2 M^{2}} H_{1,4}^{e}\left(x, 0, \vec{k}_{T}^{2}, 0,0\right)\right] \\
& =\int d^{2} \vec{k}_{T}\left[h_{1 T}\left(x, \vec{k}_{T}^{2}\right)+\frac{\vec{k}_{T}^{2}}{2 M^{2}} h_{1 T}^{\perp}\left(x, \vec{k}_{T}^{2}\right)\right], \\
H_{2}(x, 0,0) & =\int d^{2} \vec{k}_{T} E_{2,1}^{e}\left(x, 0, \vec{k}_{T}^{2}, 0,0\right)=\int d^{2} \vec{k}_{T} e\left(x, \vec{k}_{T}^{2}\right), \\
\tilde{H}_{2}^{\prime}(x, 0,0) & =\int d^{2} \vec{k}_{T} H_{2,8}^{e}\left(x, 0, \vec{k}_{T}^{2}, 0,0\right)=\int d^{2} \vec{k}_{T} h_{L}\left(x, \vec{k}_{T}^{2}\right), \\
H_{2 T}^{\prime}(x, 0,0) & =\int d^{2} \vec{k}_{T}\left[G_{2,3}^{e}\left(x, 0, \vec{k}_{T}^{2}, 0,0\right)+\frac{\vec{k}_{T}^{2}}{2 M^{2}} G_{2,4}^{e}\left(x, 0, \vec{k}_{T}^{2}, 0,0\right)\right] \\
& =\int d^{2} \vec{k}_{T}\left[g_{T}^{\prime}\left(x, \vec{k}_{T}^{2}\right)+\frac{\vec{k}_{T}^{2}}{2 M^{2}} g_{T}^{\perp}\left(x, \vec{k}_{T}^{2}\right)\right], \\
H_{3}(x, 0,0) & =\int d^{2} \vec{k}_{T} F_{3,1}^{e}\left(x, 0, \vec{k}_{T}^{2}, 0,0\right)=\int d^{2} \vec{k}_{T} f_{3}\left(x, \vec{k}_{T}^{2}\right), \\
\tilde{H}_{3}(x, 0,0) & =\int d^{2} \vec{k}_{T} G_{3,4}^{e}\left(x, 0, \vec{k}_{T}^{2}, 0,0\right)=\int d^{2} \vec{k}_{T} g_{3 L}\left(x, \vec{k}_{T}^{2}\right), \\
H_{3 T}(x, 0,0) & =\int d^{2} \vec{k}_{T}\left[H_{3,3}^{e}\left(x, 0, \vec{k}_{T}^{2}, 0,0\right)+\frac{\vec{k}_{T}^{2}}{2 M^{2}} H_{3,4}^{e}\left(x, 0, \vec{k}_{T}^{2}, 0,0\right)\right] \\
& \left.=\int h_{3 T}\left(x, \vec{k}_{T}^{2}\right)+\frac{\vec{k}_{T}^{2}}{2 M^{2}} h_{3 T}^{\perp}\left(x, \vec{k}_{T}^{2}\right)\right] .
\end{aligned}
$$

These formulas can be considered as trivial model-independent relations between GPDs and TMDs (called relations of first type in ref. 24]). Of course, the twist-2 relations (4.81)(4.83) were already known before.

Here, we are mainly interested in nontrivial relations between GPDs and TMDs that have been suggested in the literature $[18,19,20,21,22,23,24,25]$. So far explicit relations have only been established in low-order calculations in the framework of simple spectator models [20, 22, 23, 24], and in one case in a light-cone constituent quark model [25]. Our GTMD-analysis can now shed light on the question if model-independent nontrivial relations exist.

A complete classification of the nontrivial relations between GPDs and TMDs in leading twist has been performed in [24], where explicit formulae have been obtained in the same diquark spectator model as discussed in appendix C. In that work two distinct types of nontrivial relations between quark distributions have been considered - one connecting certain GPDs with the T-odd ${ }^{2}$ Sivers function $f_{1 T}^{\perp}$ [26, 27] and the Boer-Mulders function

\footnotetext{
${ }^{2}$ Note that in order to generate T-odd TMDs one has to take into account rescattering effects between
} 


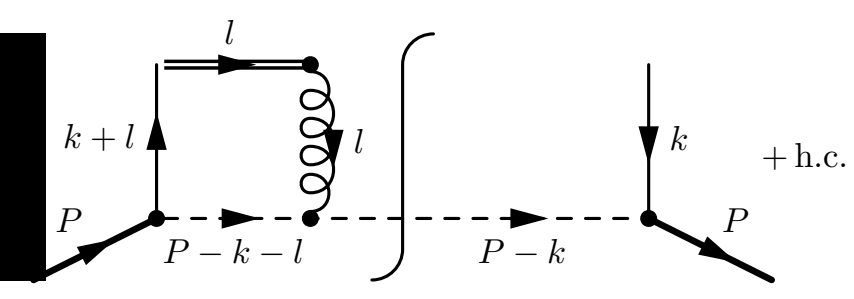

Figure 2: Lowest nontrivial order diagram for T-odd TMDs in the scalar diquark spectator model. The Hermitian conjugate diagram (h.c.) is not shown. The eikonal propagator arising from the Wilson line in the operator definition of TMDs is indicated by a double line.

$h_{1}^{\perp}$ [56] (called relations of second type in ref. [24]),

$$
\begin{aligned}
E\left(x, 0,-\vec{\Delta}_{T}^{2}\right) & \leftrightarrow-f_{1 T}^{\perp}\left(x, \vec{k}_{T}^{2} ; \eta\right), \\
E_{T}\left(x, 0,-\vec{\Delta}_{T}^{2}\right)+2 \tilde{H}_{T}\left(x, 0,-\vec{\Delta}_{T}^{2}\right) & \leftrightarrow-h_{1}^{\perp}\left(x, \vec{k}_{T}^{2} ; \eta\right),
\end{aligned}
$$

and one connecting a GPD and the T-even pretzelosity TMD $h_{1 T}^{\perp}$ (called relation of third type in ref. [24]),

$$
\tilde{H}_{T}\left(x, 0,-\vec{\Delta}_{T}^{2}\right) \leftrightarrow \frac{1}{2} h_{1 T}^{\perp}\left(x, \vec{k}_{T}^{2}\right) .
$$

As we discuss in the following our GTMD-analysis, however, does not support a modelindependent status of any such relations.

For the relations of second type in eqs. (4.90) and (4.91) this is obvious because, according to eqs. (4.4), (4.7), (4.48), (4.52), and (4.53) the involved GPDs and TMDs have different, independent mother distributions. In particular, the GPDs are connected to T-even parts of GTMDs while the TMDs are connected to T-odd parts of GTMDs. Unless, for some reason, the GTMDs are subject to further constraints one has to conclude that there cannot exist a model-independent relation between the GPDs and TMDs given in eqs. (4.90) and (4.91). This conclusion is in accordance with the observation made in [24] that nontrivial relations of second type are likely to even break down in spectator models once higher order contributions are taken into account. Therefore, one has to attribute the relations to the simplicity of the used model. Nevertheless, it may well be that numerically the model-dependent nontrivial relations work reasonably well when comparing to experimental data. In fact such a case is already known for distributions of the nucleon, namely the relation between the Sivers function and the GPD $E[18,19,20,24]$.

For the relation of third type in eq. (4.92) the GPD as well as the TMD are, according to eqs. 4.10) and (4.53), related to T-even parts of GTMDs. But the linear combinations of GTMDs differ in both cases such that no model-independent nontrivial relation of the type (4.92) can exist. In the context of the diquark spectator model the explicit relation

$$
\frac{3}{(1-x)^{2}} \tilde{H}_{T}(x, 0,0)=\int d^{2} \vec{k}_{T} h_{1 T}^{\perp}\left(x, \vec{k}_{T}^{2}\right)
$$

the active parton and the spectator system. Therefore, in the diquark spectator model the lowest order contribution to T-odd TMDs comes from the diagram shown in figure 2 . 
was established [24]. One may wonder if, in general, the specific kinematical point $\vec{\Delta}_{T}^{2}=$ $\xi=0$ and the $k_{T}$-integration used in (4.93) might spoil the above argument about different linear combinations of GTMDs. However, by taking all known symmetry properties of the GTMDs into account one is still left with such different linear combinations. Even in the simple diquark spectator model this is the case, and the relation (4.93) also just holds due to the simplicity of the model.

In order to illustrate this point we calculate the involved GPD $\tilde{H}_{T}$ and TMD $h_{1 T}^{\perp}$ in the scalar diquark model and try to preserve their respective GTMD structure as far as possible. By inserting the model results for the GTMDs in appendix Cinto eq. 4.53) one finds for the GPD $\tilde{H}_{T}$ in the case $\xi=0$

$$
\begin{aligned}
& \tilde{H}_{T}\left(x, 0,-\vec{\Delta}_{T}^{2}\right) \\
& \quad=\int d^{2} \vec{k}_{T} \tilde{C}\left[\tilde{H}_{1,2}^{e}(x)-2\left(\frac{2\left(\vec{k}_{T} \cdot \vec{\Delta}_{T}\right)^{2}-\vec{k}_{T}^{2} \vec{\Delta}_{T}^{2}}{\left(\vec{\Delta}_{T}^{2}\right)^{2}} \tilde{H}_{1,4}^{e}(x)+\tilde{H}_{1,6}^{e}(x)\right)\right] .
\end{aligned}
$$

Here we have extracted all dependence on the vectors $\vec{k}_{T}$ and $\vec{\Delta}_{T}$ from the GTMDs and put it either into their coefficients or into the overall factor

$$
\tilde{C}=\frac{g^{2}(1-x)}{2(2 \pi)^{3}} \frac{1}{\left[\left(\vec{k}_{T}+\frac{1}{2}(1-x) \vec{\Delta}_{T}\right)^{2}+\tilde{M}^{2}(x)\right]\left[\left(\vec{k}_{T}-\frac{1}{2}(1-x) \vec{\Delta}_{T}\right)^{2}+\tilde{M}^{2}(x)\right]},
$$

with

$$
\tilde{M}^{2}(x)=x m_{s}^{2}+(1-x) m_{q}^{2}-x(1-x) M^{2} .
$$

Therefore, the remnants of the GTMDs

$$
\begin{aligned}
& \tilde{H}_{1,2}^{e}(x)=(1-x)\left(m_{q}+x M\right) M, \\
& \tilde{H}_{1,4}^{e}(x)=-2 M^{2}, \\
& \tilde{H}_{1,6}^{e}(x)=\frac{1}{2}(1-x)\left(m_{q}+M\right) M
\end{aligned}
$$

only depend on the momentum fraction $x$. This allows one to perform the $\vec{k}_{T}$ integration, which yields

$$
\begin{aligned}
& \tilde{H}_{T}\left(x, 0,-\vec{\Delta}_{T}^{2}\right) \\
& \quad=\frac{g^{2}(1-x)}{8(2 \pi)^{2}} \int_{0}^{1} d \alpha \frac{2 \tilde{H}_{1,2}^{e}(x)-(1-2 \alpha)^{2}(1-x)^{2} \tilde{H}_{1,4}^{e}(x)-4 \tilde{H}_{1,6}^{e}(x)}{\alpha(1-\alpha)(1-x)^{2} \vec{\Delta}_{T}^{2}+\tilde{M}^{2}(x)} .
\end{aligned}
$$

In the forward limit this leads to

$$
\tilde{H}_{T}(x, 0,0)=\frac{g^{2}(1-x)}{8(2 \pi)^{2}} \frac{2 \tilde{H}_{1,2}^{e}(x)-\frac{1}{3}(1-x)^{2} \tilde{H}_{1,4}^{e}(x)-4 \tilde{H}_{1,6}^{e}(x)}{\tilde{M}^{2}(x)} .
$$

On the other hand, one finds for the zeroth moment of the TMD $h_{1 T}^{\perp}$ by inserting the model results for the GTMDs in appendix C into eq. (4.10)

$$
\int d^{2} \vec{k}_{T} h_{1 T}^{\perp}\left(x, \vec{k}_{T}^{2}\right)=\frac{g^{2}(1-x)}{4(2 \pi)^{2}} \frac{\tilde{H}_{1,4}^{e}(x)}{\tilde{M}^{2}(x)} .
$$


This shows explicitly that the GPD $\tilde{H}_{T}$ and the TMD $h_{1 T}^{\perp}$ are connected to different remnants of GTMDs even in the scalar diquark model.

However, due to the simplicity of the scalar diquark model the remnants of the GTMDs are related according to

$$
2 \tilde{H}_{1,2}^{e}(x)-4 \tilde{H}_{1,6}^{e}(x)=-2(1-x)^{2} M^{2}=(1-x)^{2} \tilde{H}_{1,4}^{e}(x)
$$

This immediately implies the relation

$$
\frac{3}{(1-x)^{2}} \tilde{H}_{T}(x, 0,0)=\frac{g^{2}(1-x)}{4(2 \pi)^{2}} \frac{\tilde{H}_{1,4}^{e}(x)}{\tilde{M}^{2}(x)}=\int d^{2} \vec{k}_{T} h_{1 T}^{\perp}\left(x, \vec{k}_{T}^{2}\right),
$$

which we already quoted above in (4.93). It should be stressed once again, that this relation only holds due to the simplicity of the scalar diquark model. In general, no dependence like eq. (4.103) will exist between the different, independent GTMDs. We note that a relation like (4.93) was also obtained in a specific light-cone quark model [25], but in that model a factor different from 3 on the l.h.s. of (4.93) shows up ${ }^{3}$. The fact that a formula corresponding to (4.93) emerges in the framework of another model does not contradict our general argument that in full QCD a relation of the type (4.92) cannot hold.

By extending our GTMD analysis we find that also for twist-3 and twist-4 no modelindependent nontrivial relations between GPDs and TMDs exist. On the other hand such relations may well emerge in the framework of simple models.

\section{Conclusions}

In summary, we have derived the structure of the fully unintegrated, off-diagonal quarkquark correlator for a spin- $1 / 2$ hadron, and thus extended our previous study of the spin-0 case [1]. This object, which contains the most general information on the two-parton structure of a hadron, has been parameterized in terms of so-called generalized parton correlation functions (GPCFs). The major challenge in this derivation was to eliminate all redundant terms without missing any relevant term at the same time. Integrating the GPCFs upon a light-cone component of the quark momentum one ends up with entities which we called generalized transverse momentum dependent parton distributions (GTMDs). In general, GTMDs can be of direct relevance for the phenomenology of various hard (diffractive) processes (see, e.g., refs. 36, 37, 35, 38]). Our analysis shows that both the GPCFs and the GTMDs in general are complex-valued functions. This is different from the (simpler) forward parton distributions, GPDs, and TMDs all of which are real.

Suitable projections of GTMDs lead to GPDs on the one hand and TMDs on the other. Therefore, GTMDs can be considered as mother distributions of GPDs and TMDs [16, 17, [10]. To study these two limiting cases of GTMDs was the main motivation of the present work. One outcome was the first complete classification of GPDs for a spin-1/2 hadron beyond leading twist. Most importantly, we were able to determine which of the GPDs

\footnotetext{
${ }^{3}$ Actually, in ref. 25] the factor 3 appeared, but later on an error in the calculation was found [57].
} 
and TMDs have the same mother distributions allowing us to explore whether modelindependent relations between GPDs and TMDs can be established. One ends up with nine such model-independent relations. Actually, these cases can be considered as trivial ones because the respective GPDs and TMDs also have a relation to the same forward parton distributions (see also ref. [24]). Our main interest was to investigate nontrivial relations between GPDs and TMDs which have been obtained in models and extensively discussed in the recent literature $[18,19,20,21,22,23,24,25]$. We have restricted this study to leading twist where three nontrivial relations have been found (see 24 for an overview) - two involving the T-odd Sivers TMD $f_{1 T}^{\perp}$ and the Boer-Mulders TMD $h_{1}^{\perp}$, and one in which the T-even pretzelosity TMD $h_{1 T}^{\perp}$ shows up. It turns out that none of these relations can be promoted to a model-independent status as the respective functions are related to different (linear combinations of) GTMDs. For the relations containing Todd TMDs this finding agrees with ref. [24] where it has been argued that these nontrivial relations between GPDs and TMDs are likely to break down even in spectator models if the parton distributions are evaluated to higher order in perturbation theory. Moreover, our model-independent study for the Boer-Mulders function of a spin-0 hadron came to the same conclusion [1]. We emphasize that our finding does not tell anything about the numerical violation of (model-dependent) nontrivial relations between GPDs and TMDs. On the other hand, such relations have hardly any predictive power and only after all the involved distributions have been measured one can really judge about their quality.

\section{Acknowledgments}

The work has partially been supported by the Verbundforschung "Hadronen und Kerne" of the BMBF and by the Deutsche Forschungsgemeinschaft (DFG).

Notice: Authored by Jefferson Science Associates, LLC under U.S. DOE Contract No. DE-AC05-06OR23177. The U.S. Government retains a non-exclusive, paid-up, irrevocable, world-wide license to publish or reproduce this manuscript for U.S. Government purposes.

\section{A. Parameterization of Dirac bilinears}

In this appendix we derive the most general parameterization of the scalar, pseudoscalar, vector, axial vector, and tensor Dirac bilinear introduced in the eqs. (2.6)-(2.10) respecting the corresponding constraints from parity

$$
\begin{aligned}
\Gamma_{\mathrm{S}}(P, k, \Delta, N ; \eta) & =+\gamma_{0} \Gamma_{\mathrm{S}}(\bar{P}, \bar{k}, \bar{\Delta}, \bar{N} ; \eta) \gamma_{0}, \\
\Gamma_{\mathrm{P}}(P, k, \Delta, N ; \eta) & =-\gamma_{0} \Gamma_{\mathrm{P}}(\bar{P}, \bar{k}, \bar{\Delta}, \bar{N} ; \eta) \gamma_{0}, \\
\Gamma_{\mathrm{V}}^{\mu}(P, k, \Delta, N ; \eta) & =+\gamma_{0} \Gamma_{\mathrm{V}}^{\bar{\mu}}(\bar{P}, \bar{k}, \bar{\Delta}, \bar{N} ; \eta) \gamma_{0}, \\
\Gamma_{\mathrm{A}}^{\mu}(P, k, \Delta, N ; \eta) & =-\gamma_{0} \Gamma_{\mathrm{A}}^{\bar{\mu}}(\bar{P}, \bar{k}, \bar{\Delta}, \bar{N} ; \eta) \gamma_{0}, \\
\Gamma_{\mathrm{T}}^{\mu \nu}(P, k, \Delta, N ; \eta) & =+\gamma_{0} \Gamma_{\mathrm{T}}^{\bar{\mu} \bar{\nu}}(\bar{P}, \bar{k}, \bar{\Delta}, \bar{N} ; \eta) \gamma_{0}
\end{aligned}
$$

For this purpose we generalize the method described in [54] for GPDs in an appropriate way to the case of GPCFs. In order to eliminate independent terms in the parameterization 
of the Dirac bilinears we use the Gordon identities

$$
\begin{aligned}
\bar{u}\left(p^{\prime}, \lambda^{\prime}\right) \gamma^{\mu} u(p, \lambda) & =\bar{u}\left(p^{\prime}, \lambda^{\prime}\right)\left[\frac{P^{\mu}}{M}+\frac{i \sigma^{\mu \Delta}}{2 M}\right] u(p, \lambda), \\
0 & =\bar{u}\left(p^{\prime}, \lambda^{\prime}\right)\left[\frac{\Delta^{\mu}}{2 M}+\frac{i \sigma^{\mu P}}{M}\right] u(p, \lambda), \\
\bar{u}\left(p^{\prime}, \lambda^{\prime}\right) \gamma^{\mu} \gamma_{5} u(p, \lambda) & =\bar{u}\left(p^{\prime}, \lambda^{\prime}\right)\left[\frac{\Delta^{\mu} \gamma_{5}}{2 M}+\frac{i \sigma^{\mu P} \gamma_{5}}{M}\right] u(p, \lambda), \\
0 & =\bar{u}\left(p^{\prime}, \lambda^{\prime}\right)\left[\frac{P^{\mu} \gamma_{5}}{M}+\frac{i \sigma^{\mu \Delta} \gamma_{5}}{2 M}\right] u(p, \lambda) .
\end{aligned}
$$

In addition, we also use the $\varepsilon$-identity

$$
g^{\alpha \beta} \varepsilon^{\mu \nu \rho \sigma}=g^{\mu \beta} \varepsilon^{\alpha \nu \rho \sigma}+g^{\nu \beta} \varepsilon^{\mu \alpha \rho \sigma}+g^{\rho \beta} \varepsilon^{\mu \nu \alpha \sigma}+g^{\sigma \beta} \varepsilon^{\mu \nu \rho \alpha}
$$

as well as the $\sigma$-identity

$$
i \sigma^{\mu \nu} \gamma_{5}=-\frac{1}{2} \varepsilon^{\mu \nu \rho \sigma} \sigma_{\rho \sigma}
$$

\section{A.1 Parameterization of the scalar Dirac bilinear}

A complete parameterization of the scalar Dirac bilinear in eq. (2.6) can be obtained by treating all possible Dirac currents one after the other:

1. vector current $\left[\bar{u}\left(p^{\prime}, \lambda^{\prime}\right) \gamma^{\mu} u(p, \lambda)\right]$ : Using the Gordon identity in eq. (A.6) all vector currents can be replaced by scalar and tensor currents.

2. axial vector current $\left[\bar{u}\left(p^{\prime}, \lambda^{\prime}\right) \gamma^{\mu} \gamma_{5} u(p, \lambda)\right]$ : Using the Gordon identity in eq. (A.8) all axial vector currents can be replaced by pseudoscalar and pseudotensor currents.

3. pseudoscalar current $\left[\bar{u}\left(p^{\prime}, \lambda^{\prime}\right) \gamma_{5} u(p, \lambda)\right]$ : Contracting the Gordon identity in eq. (A.9) with the light-cone vector $N$ yields

$$
\bar{u}\left(p^{\prime}, \lambda^{\prime}\right) \gamma_{5} u(p, \lambda)=\bar{u}\left(p^{\prime}, \lambda^{\prime}\right) \frac{i \sigma^{\Delta N} \gamma_{5}}{2 M^{2}} u(p, \lambda),
$$

so that all pseudoscalar currents can be replaced by pseudotensor currents.

4. pseudotensor current $\left[\bar{u}\left(p^{\prime}, \lambda^{\prime}\right) i \sigma^{\mu \nu} \gamma_{5} u(p, \lambda)\right]$ : Using the $\sigma$-identity in eq. (A.11) all pseudotensor currents can be replaced by tensor currents.

5. tensor current $\left[\bar{u}\left(p^{\prime}, \lambda^{\prime}\right) i \sigma^{\mu \nu} u(p, \lambda)\right]$ : All possible tensor currents are of the form

$$
\bar{u}\left(p^{\prime}, \lambda^{\prime}\right) i \sigma^{a b} u(p, \lambda)
$$

with $a$ and $b$ being any of the vectors $P, k, \Delta$, and $N$. Using the Gordon identity in eq. (A.7) all tensor currents containing the vector $P$ contracted with $\sigma$ can be replaced by scalar currents. Therefore, one is left with three tensor currents

$$
\bar{u}\left(p^{\prime}, \lambda^{\prime}\right) i \sigma^{k \Delta} u(p, \lambda), \quad \bar{u}\left(p^{\prime}, \lambda^{\prime}\right) i \sigma^{k N} u(p, \lambda), \quad \bar{u}\left(p^{\prime}, \lambda^{\prime}\right) i \sigma^{\Delta N} u(p, \lambda) .
$$


6. scalar current $\left[\bar{u}\left(p^{\prime}, \lambda^{\prime}\right) u(p, \lambda)\right]$ : There is only one possible scalar current

$$
\bar{u}\left(p^{\prime}, \lambda^{\prime}\right) u(p, \lambda)
$$

which can not be replaced.

To summarize, the scalar Dirac bilinear in eq. (2.6) is completely parameterized by the four currents in eqs. (A.14) and (A.15), i. e.,

$$
\begin{aligned}
& \bar{u}\left(p^{\prime}, \lambda^{\prime}\right) \Gamma_{\mathrm{S}}(P, k, \Delta, N ; \eta) u(p, \lambda) \\
& \quad=\bar{u}\left(p^{\prime}, \lambda^{\prime}\right)\left[A_{1}^{E}+\frac{i \sigma^{k \Delta}}{M^{2}} A_{2}^{E}+\frac{i \sigma^{k N}}{M^{2}} A_{3}^{E}+\frac{i \sigma^{\Delta N}}{M^{2}} A_{4}^{E}\right] u(p, \lambda),
\end{aligned}
$$

where the GPCFs $A_{n}^{E}$ are scalar functions of $P, k, \Delta, N$, and $\eta$.

\section{A.2 Parameterization of the pseudoscalar Dirac bilinear}

A complete parameterization of the pseudoscalar Dirac bilinear in eq. (2.7) can be obtained by treating all possible Dirac currents one after the other:

1. vector current $\left[\bar{u}\left(p^{\prime}, \lambda^{\prime}\right) \gamma^{\mu} u(p, \lambda)\right]$ : Using the Gordon identity in eq. (A.6) all vector currents can be replaced by scalar and tensor currents.

2. axial vector current $\left[\bar{u}\left(p^{\prime}, \lambda^{\prime}\right) \gamma^{\mu} \gamma_{5} u(p, \lambda)\right]$ : Using the Gordon identity in eq. (A.8) all axial vector currents can be replaced by pseudoscalar and pseudotensor currents.

3. pseudoscalar current $\left[\bar{u}\left(p^{\prime}, \lambda^{\prime}\right) \gamma_{5} u(p, \lambda)\right]$ : Using eq. (A.12) all pseudoscalar currents can be replaced by pseudotensor currents.

4. tensor current $\left[\bar{u}\left(p^{\prime}, \lambda^{\prime}\right) i \sigma^{\mu \nu} u(p, \lambda)\right]$ : Using the $\sigma$-identity in eq. (A.11) all tensor currents can be replaced by pseudotensor currents.

5. pseudotensor current $\left[\bar{u}\left(p^{\prime}, \lambda^{\prime}\right) i \sigma^{\mu \nu} \gamma_{5} u(p, \lambda)\right]$ : All possible pseudotensor currents are of the form

$$
\bar{u}\left(p^{\prime}, \lambda^{\prime}\right) i \sigma^{a b} \gamma_{5} u(p, \lambda)
$$

with $a$ and $b$ being any of the vectors $P, k, \Delta$, and $N$. Now, multiplying the Gordon identity in eq. (A.7) with an $\varepsilon$-tensor and using the $\varepsilon$-identity in eq. (A.10) as well as the $\sigma$-identity in eq. (A.11) yields

$$
0=\bar{u}\left(p^{\prime}, \lambda^{\prime}\right)\left[\frac{P^{\mu} i \sigma^{\nu \rho} \gamma_{5}}{M}+\frac{P^{\nu} i \sigma^{\rho \mu} \gamma_{5}}{M}+\frac{P^{\rho} i \sigma^{\mu \nu} \gamma_{5}}{M}-\frac{i \varepsilon^{\mu \nu \rho \Delta}}{2 M}\right] u(p, \lambda)
$$


Contracting this equation in turn with $P_{\mu}, k_{\nu}, N_{\rho}$ and $P_{\mu}, \Delta_{\nu}, N_{\rho}$ and $k_{\mu}, \Delta_{\nu}, N_{\rho}$ allows to replace some pseudotensor currents by scalar and pseudotensor currents

$$
\begin{aligned}
& \bar{u}\left(p^{\prime}, \lambda^{\prime}\right) i \sigma^{P k} \gamma_{5} u(p, \lambda) \\
& \quad=\bar{u}\left(p^{\prime}, \lambda^{\prime}\right)\left[\frac{P \cdot k i \sigma^{P N} \gamma_{5}}{M^{2}}-\frac{P^{2} i \sigma^{k N} \gamma_{5}}{M^{2}}-\frac{i \varepsilon^{P k \Delta N}}{2 M^{2}}\right] u(p, \lambda), \\
& \bar{u}\left(p^{\prime}, \lambda^{\prime}\right) i \sigma^{P \Delta} \gamma_{5} u(p, \lambda) \\
& \quad=\bar{u}\left(p^{\prime}, \lambda^{\prime}\right)\left[-\frac{P^{2} i \sigma^{\Delta N} \gamma_{5}}{M^{2}}\right] u(p, \lambda), \\
& \bar{u}\left(p^{\prime}, \lambda^{\prime}\right) i \sigma^{k \Delta} \gamma_{5} u(p, \lambda) \\
& \quad=\bar{u}\left(p^{\prime}, \lambda^{\prime}\right)\left[-\frac{P \cdot k i \sigma^{\Delta N} \gamma_{5}}{M^{2}}\right] u(p, \lambda),
\end{aligned}
$$

so that one is left with three pseudotensor currents

$$
\bar{u}\left(p^{\prime}, \lambda^{\prime}\right) i \sigma^{P N} \gamma_{5} u(p, \lambda), \quad \bar{u}\left(p^{\prime}, \lambda^{\prime}\right) i \sigma^{k N} \gamma_{5} u(p, \lambda), \quad \bar{u}\left(p^{\prime}, \lambda^{\prime}\right) i \sigma^{\Delta N} \gamma_{5} u(p, \lambda) .
$$

6. scalar current $\left[\bar{u}\left(p^{\prime}, \lambda^{\prime}\right) u(p, \lambda)\right]$ : There is only one possible scalar current

$$
\bar{u}\left(p^{\prime}, \lambda^{\prime}\right) i \varepsilon^{P k \Delta N} u(p, \lambda),
$$

which can not be replaced.

To summarize, the pseudoscalar Dirac bilinear in eq. (2.7) is completely parameterized by the four currents in eqs. (A.22) and (A.23), i. e.,

$$
\begin{aligned}
& \bar{u}\left(p^{\prime}, \lambda^{\prime}\right) \Gamma_{\mathrm{P}}(P, k, \Delta, N ; \eta) u(p, \lambda) \\
& \quad=\bar{u}\left(p^{\prime}, \lambda^{\prime}\right)\left[\frac{i \varepsilon^{P k \Delta N}}{M^{4}} A_{5}^{E}+\frac{i \sigma^{P N} \gamma_{5}}{M^{2}} A_{6}^{E}+\frac{i \sigma^{k N} \gamma_{5}}{M^{2}} A_{7}^{E}+\frac{i \sigma^{\Delta N} \gamma_{5}}{M^{2}} A_{8}^{E}\right] u(p, \lambda),
\end{aligned}
$$

where the GPCFs $A_{n}^{E}$ are scalar functions of $P, k, \Delta, N$, and $\eta$.

\section{A.3 Parameterization of the vector Dirac bilinear}

A complete parameterization of the vector Dirac bilinear in eq. (2.8) can be obtained by treating all possible Dirac currents one after the other:

1. vector current $\left[\bar{u}\left(p^{\prime}, \lambda^{\prime}\right) \gamma^{\mu} u(p, \lambda)\right]$ : Using the Gordon identity in eq. (A.6) all vector currents can be replaced by scalar and tensor currents.

2. axial vector current $\left[\bar{u}\left(p^{\prime}, \lambda^{\prime}\right) \gamma^{\mu} \gamma_{5} u(p, \lambda)\right]$ : Using the Gordon identity in eq. (A.8) all axial vector currents can be replaced by pseudoscalar and pseudotensor currents.

3. pseudoscalar current $\left[\bar{u}\left(p^{\prime}, \lambda^{\prime}\right) \gamma_{5} u(p, \lambda)\right]$ : Using eq. (A.12) all pseudoscalar currents can be replaced by pseudotensor currents.

4. pseudotensor current $\left[\bar{u}\left(p^{\prime}, \lambda^{\prime}\right) i \sigma^{\mu \nu} \gamma_{5} u(p, \lambda)\right]$ : Using the $\sigma$-identity in eq. (A.11) all pseudotensor currents can be replaced by tensor currents. 
5. tensor current $\left[\bar{u}\left(p^{\prime}, \lambda^{\prime}\right) i \sigma^{\mu \nu} u(p, \lambda)\right]$ : All possible tensor currents are of the form

$$
\bar{u}\left(p^{\prime}, \lambda^{\prime}\right) i \sigma^{\mu a} u(p, \lambda), \quad \bar{u}\left(p^{\prime}, \lambda^{\prime}\right) a^{\mu} i \sigma^{b c} u(p, \lambda)
$$

with $a, b$, and $c$ being any of the vectors $P, k, \Delta$, and $N$. Using the Gordon identity in eq. (A.7) all tensor currents containing the vector $P$ contracted with $\sigma$ can be replaced by scalar currents. Therefore, one is left with 15 tensor currents

$$
\begin{array}{lll}
\bar{u}\left(p^{\prime}, \lambda^{\prime}\right) i \sigma^{\mu k} u(p, \lambda), & \bar{u}\left(p^{\prime}, \lambda^{\prime}\right) i \sigma^{\mu \Delta} u(p, \lambda), & \bar{u}\left(p^{\prime}, \lambda^{\prime}\right) i \sigma^{\mu N} u(p, \lambda), \\
\bar{u}\left(p^{\prime}, \lambda^{\prime}\right) a^{\mu} i \sigma^{k \Delta} u(p, \lambda), & \bar{u}\left(p^{\prime}, \lambda^{\prime}\right) a^{\mu} i \sigma^{k N} u(p, \lambda), & \bar{u}\left(p^{\prime}, \lambda^{\prime}\right) a^{\mu} i \sigma^{\Delta N} u(p, \lambda)
\end{array}
$$

with $a$ being any of the vectors $P, k, \Delta$, and $N$.

6. scalar current $\left[\bar{u}\left(p^{\prime}, \lambda^{\prime}\right) u(p, \lambda)\right]$ : There are four possible scalar currents

$$
\bar{u}\left(p^{\prime}, \lambda^{\prime}\right) a^{\mu} u(p, \lambda)
$$

with $a$ being any of the vectors $P, k, \Delta$, and $N$, which can not be replaced.

So far, we were able to reduce the number of currents needed to parameterize the vector Dirac bilinear in eq. (2.8) to the 19 currents in eqs. (A.26) and (A.27). However, it is possible to reduce this number even further by using that in Minkowski space

$$
\operatorname{det}\left(\begin{array}{ccccc}
g^{\alpha \mu} & g^{\beta \mu} & g^{\gamma \mu} & g^{\delta \mu} & g^{\varepsilon \mu} \\
g^{\alpha \nu} & g^{\beta \nu} & g^{\gamma \nu} & g^{\delta \nu} & g^{\varepsilon \nu} \\
g^{\alpha \rho} & g^{\beta \rho} & g^{\gamma \rho} & g^{\delta \rho} & g^{\varepsilon \rho} \\
g^{\alpha \sigma} & g^{\beta \sigma} & g^{\gamma \sigma} & g^{\delta \sigma} & g^{\varepsilon \sigma} \\
g^{\alpha \tau} & g^{\beta \tau} & g^{\gamma \tau} & g^{\delta \tau} & g^{\varepsilon \tau}
\end{array}\right)=0
$$

Contracting this determinant with $P_{\alpha}, k_{\beta}, \Delta_{\gamma}, N_{\delta}, P_{\nu}, k_{\rho}, \Delta_{\sigma}$, and $N_{\tau}$ and then in turn with $i \sigma_{\varepsilon}{ }^{k}, i \sigma_{\varepsilon}{ }^{\Delta}$, and $i \sigma_{\varepsilon}{ }^{N}$ yields

$$
\begin{gathered}
\bar{u}\left(p^{\prime}, \lambda^{\prime}\right) \operatorname{det}\left(\begin{array}{ccccc}
P^{\mu} & k^{\mu} & \Delta^{\mu} & N^{\mu} & i \sigma^{\mu k} \\
P^{2} & P \cdot k & 0 & P \cdot N & \frac{1}{2} k \cdot \Delta \\
P \cdot k & k^{2} & k \cdot \Delta & k \cdot N & 0 \\
0 & k \cdot \Delta & \Delta^{2} & \Delta \cdot N & -i \sigma^{k \Delta} \\
P \cdot N & k \cdot N & \Delta \cdot N & 0 & -i \sigma^{k N}
\end{array}\right) u(p, \lambda)=0, \\
\bar{u}\left(p^{\prime}, \lambda^{\prime}\right) \operatorname{det}\left(\begin{array}{ccccc}
P^{\mu} & k^{\mu} & \Delta^{\mu} & N^{\mu} & i \sigma^{\mu \Delta} \\
P^{2} & P \cdot k & 0 & P \cdot N & \frac{1}{2} \Delta^{2} \\
P \cdot k & k^{2} & k \cdot \Delta & k \cdot N & i \sigma^{k \Delta} \\
0 & k \cdot \Delta & \Delta^{2} & \Delta \cdot N & 0 \\
P \cdot N & k \cdot N & \Delta \cdot N & 0 & -i \sigma^{\Delta N}
\end{array}\right) u(p, \lambda)=0, \\
\bar{u}\left(p^{\prime}, \lambda^{\prime}\right) \operatorname{det}\left(\begin{array}{ccccc}
P^{\mu} & k^{\mu} & \Delta^{\mu} & N^{\mu} & i \sigma^{\mu N} \\
P^{2} & P \cdot k & 0 & P \cdot N & \frac{1}{2} \Delta \cdot N \\
P \cdot k & k^{2} & k \cdot \Delta & k \cdot N & i \sigma^{k N} \\
0 & k \cdot \Delta & \Delta^{2} & \Delta \cdot N & i \sigma^{\Delta N} \\
P \cdot N & k \cdot N & \Delta \cdot N & 0 & 0
\end{array}\right) u(p, \lambda)=0 .
\end{gathered}
$$


For $P \neq 0, k \neq 0, \Delta \neq 0$, and $N \neq 0$ these three equations are non-trivial and allow one to eliminate three of the 19 currents we have left. As $P$ and $N$ are always different from zero, it is most convenient to eliminate the tensor currents

$$
\Delta^{\mu} i \sigma^{k \Delta}, \quad \Delta^{\mu} i \sigma^{k N}, \quad k^{\mu} i \sigma^{\Delta N}
$$

because either $k$ and $\Delta$ are different form zero and we are able to eliminate them using the constraints in eqs. (A.29)-(A.31) or at least one of the vectors is zero and the whole current vanishes. Therefore, one possible parameterization of the vector Dirac bilinear in eq. (2.8), is given by

$$
\begin{aligned}
\bar{u}\left(p^{\prime}, \lambda^{\prime}\right) \Gamma_{\mathrm{V}}^{\mu}(P, k, \Delta, N ; \eta) u(p, \lambda) & \\
= & \bar{u}\left(p^{\prime}, \lambda^{\prime}\right)\left[\frac{P^{\mu}}{M} A_{1}^{F}+\frac{k^{\mu}}{M} A_{2}^{F}+\frac{\Delta^{\mu}}{M} A_{3}^{F}+\frac{N^{\mu}}{M} A_{4}^{F}+\frac{i \sigma^{\mu k}}{M} A_{5}^{F}+\frac{i \sigma^{\mu \Delta}}{M} A_{6}^{F}+\frac{i \sigma^{\mu N}}{M} A_{7}^{F}\right. \\
& +\frac{P^{\mu} i \sigma^{k \Delta}}{M^{3}} A_{8}^{F}+\frac{k^{\mu} i \sigma^{k \Delta}}{M^{3}} A_{9}^{F}+\frac{N^{\mu} i \sigma^{k \Delta}}{M^{3}} A_{10}^{F}+\frac{P^{\mu} i \sigma^{k N}}{M^{3}} A_{11}^{F}+\frac{k^{\mu} i \sigma^{k N}}{M^{3}} A_{12}^{F} \\
& \left.+\frac{N^{\mu} i \sigma^{k N}}{M^{3}} A_{13}^{F}+\frac{P^{\mu} i \sigma^{\Delta N}}{M^{3}} A_{14}^{F}+\frac{\Delta^{\mu} i \sigma^{\Delta N}}{M^{3}} A_{15}^{F}+\frac{N^{\mu} i \sigma^{\Delta N}}{M^{3}} A_{16}^{F}\right] u(p, \lambda),
\end{aligned}
$$

where the GPCFs $A_{n}^{F}$ are scalar functions of $P, k, \Delta, N$, and $\eta$.

\section{A.4 Parameterization of the axial vector Dirac bilinear}

A complete parameterization of the axial vector Dirac bilinear in eq. (2.9) can be obtained by treating all possible Dirac currents one after the other:

1. vector current $\left[\bar{u}\left(p^{\prime}, \lambda^{\prime}\right) \gamma^{\mu} u(p, \lambda)\right]$ : Using the Gordon identity in eq. (A.6) all vector currents can be replaced by scalar and tensor currents.

2. axial vector current $\left[\bar{u}\left(p^{\prime}, \lambda^{\prime}\right) \gamma^{\mu} \gamma_{5} u(p, \lambda)\right]$ : Using the Gordon identity in eq. (A.8) all axial vector currents can be replaced by pseudoscalar and pseudotensor currents.

3. pseudoscalar current $\left[\bar{u}\left(p^{\prime}, \lambda^{\prime}\right) \gamma_{5} u(p, \lambda)\right]$ : Using eq. (A.12) all pseudoscalar currents can be replaced by pseudotensor currents.

4. tensor current $\left[\bar{u}\left(p^{\prime}, \lambda^{\prime}\right) i \sigma^{\mu \nu} u(p, \lambda)\right]$ : Using the $\sigma$-identity in eq. (A.11) all tensor currents can be replaced by pseudotensor currents.

5. pseudotensor current $\left[\bar{u}\left(p^{\prime}, \lambda^{\prime}\right) i \sigma^{\mu \nu} \gamma_{5} u(p, \lambda)\right]$ : All possible pseudotensor currents are of the form

$$
\bar{u}\left(p^{\prime}, \lambda^{\prime}\right) i \sigma^{\mu a} \gamma_{5} u(p, \lambda), \quad \bar{u}\left(p^{\prime}, \lambda^{\prime}\right) a^{\mu} i \sigma^{b c} \gamma_{5} u(p, \lambda)
$$


with $a, b$ and $c$ being any of the vectors $P, k, \Delta$, and $N$. Now, contracting eq. (A.18) with $N_{\nu}$ and in turn with $P_{\rho}, k_{\rho}$, and $\Delta_{\rho}$ yields

$$
\begin{aligned}
& \bar{u}\left(p^{\prime}, \lambda^{\prime}\right) i \sigma^{\mu P} \gamma_{5} u(p, \lambda) \\
& \quad=\bar{u}\left(p^{\prime}, \lambda^{\prime}\right)\left[\frac{P^{2} i \sigma^{\mu N} \gamma_{5}}{M^{2}}-\frac{P^{\mu} i \sigma^{P N} \gamma_{5}}{M^{2}}-\frac{i \varepsilon^{\mu P \Delta N}}{2 M^{2}}\right] u(p, \lambda), \\
& \bar{u}\left(p^{\prime}, \lambda^{\prime}\right) i \sigma^{\mu k} \gamma_{5} u(p, \lambda) \\
& \quad=\bar{u}\left(p^{\prime}, \lambda^{\prime}\right)\left[\frac{P \cdot k i \sigma^{\mu N} \gamma_{5}}{M^{2}}-\frac{P^{\mu} i \sigma^{k N} \gamma_{5}}{M^{2}}-\frac{i \varepsilon^{\mu k \Delta N}}{2 M^{2}}\right] u(p, \lambda), \\
& \bar{u}\left(p^{\prime}, \lambda^{\prime}\right) i \sigma^{\mu \Delta} \gamma_{5} u(p, \lambda) \\
& \quad=\bar{u}\left(p^{\prime}, \lambda^{\prime}\right)\left[-\frac{P^{\mu} i \sigma^{\Delta N} \gamma_{5}}{M^{2}}\right] u(p, \lambda)
\end{aligned}
$$

which together with eqs. (A.19)-(A.21) allows to replace some pseudotensor currents by scalar and pseudotensor currents. Therefore, one is left with 13 pseudotensor currents

$$
\begin{array}{lll}
\bar{u}\left(p^{\prime}, \lambda^{\prime}\right) i \sigma^{\mu P} \gamma_{5} u(p, \lambda), & \bar{u}\left(p^{\prime}, \lambda^{\prime}\right) i \sigma^{\mu k} \gamma_{5} u(p, \lambda), \quad \bar{u}\left(p^{\prime}, \lambda^{\prime}\right) i \sigma^{\mu N} \gamma_{5} u(p, \lambda), \\
\bar{u}\left(p^{\prime}, \lambda^{\prime}\right) a^{\mu} i \sigma^{P N} \gamma_{5} u(p, \lambda), & \bar{u}\left(p^{\prime}, \lambda^{\prime}\right) a^{\mu} i \sigma^{k N} \gamma_{5} u(p, \lambda), \\
\bar{u}\left(p^{\prime}, \lambda^{\prime}\right) P^{\mu} i \sigma^{\Delta N} \gamma_{5} u(p, \lambda), & \bar{u}\left(p^{\prime}, \lambda^{\prime}\right) a^{\mu} i \sigma^{\Delta N} \gamma_{5} u(p, \lambda)
\end{array}
$$

with $a$ being any of the vectors $k, \Delta$, and $N$.

6. scalar current $\left[\bar{u}\left(p^{\prime}, \lambda^{\prime}\right) u(p, \lambda)\right]$ : There are four possible scalar currents

$$
\bar{u}\left(p^{\prime}, \lambda^{\prime}\right) i \varepsilon^{\mu a b c} u(p, \lambda)
$$

with $a, b$, and $c$ being any of the vectors $P, k, \Delta$, and $N$, which can not be replaced.

So far, we were able to reduce the number of currents needed to parameterize the axial vector Dirac bilinear in eq. (2.9) to the 17 currents in eqs. (A.38) and (A.39). However, it is possible to reduce this number even further by considering eq. (A.28). Contracting this determinant with $P_{\alpha}, k_{\beta}, \Delta_{\gamma}, N_{\delta}, P_{\nu}, k_{\rho}, \Delta_{\sigma}, N_{\tau}$ and $i \sigma_{\varepsilon}{ }^{N} \gamma_{5}$ and using eqs. (A.19)-(A.21) and (A.35)-A.37) yields

$$
\begin{aligned}
& \bar{u}\left(p^{\prime}, \lambda^{\prime}\right) \operatorname{det}\left(\begin{array}{ccccc}
0 & k^{\mu} & \Delta^{\mu} & N^{\mu} & 0 \\
P^{2} & P \cdot k & 0 & P \cdot N & i \sigma^{P N} \gamma_{5} \\
P \cdot k & k^{2} & k \cdot \Delta & k \cdot N & i \sigma^{k N} \gamma_{5} \\
0 & k \cdot \Delta & \Delta^{2} & \Delta \cdot N & i \sigma^{\Delta N} \gamma_{5} \\
P \cdot N & k \cdot N & \Delta \cdot N & 0 & 0
\end{array}\right) u(p, \lambda) \\
& =\bar{u}\left(p^{\prime}, \lambda^{\prime}\right) \operatorname{det}\left(\begin{array}{cccc}
P \cdot k & 0 & P \cdot N & -P \cdot N i \sigma^{\mu P} \gamma_{5}-\frac{1}{2} i \varepsilon^{\mu P \Delta N} \\
k^{2} & k \cdot \Delta & k \cdot N & -P \cdot N i \sigma^{\mu k} \gamma_{5}-\frac{1}{2} i \varepsilon^{\mu k \Delta N} \\
k \cdot \Delta & \Delta^{2} & \Delta \cdot N & P^{\mu} i \sigma^{\Delta N} \gamma_{5} \\
k \cdot N & \Delta \cdot N & 0 & -P \cdot N i \sigma^{\mu N} \gamma_{5}
\end{array}\right) u(p, \lambda) .
\end{aligned}
$$


For $P \neq 0, k \neq 0, \Delta \neq 0$, and $N \neq 0$ this equation is non-trivial and allows one to eliminate one of the 17 currents we have left. As $P$ and $N$ are always different from zero, it is most convenient to eliminate the tensor current

$$
k^{\mu} i \sigma^{\Delta N} \gamma_{5}
$$

because either $k$ and $\Delta$ are different form zero and we are able to eliminate it using the constraint in eq. (A.40) or at least one of the vectors is zero and the whole current vanishes. Therefore, one possible parameterization of the axial vector Dirac bilinear in eq. (2.9), is given by

$$
\begin{aligned}
\bar{u}\left(p^{\prime}, \lambda^{\prime}\right) \Gamma_{\mathrm{A}}^{\mu}(P, k, \Delta, N ; \eta) u(p, \lambda) & \\
= & \bar{u}\left(p^{\prime}, \lambda^{\prime}\right)\left[\frac{i \varepsilon^{\mu P k \Delta}}{M^{3}} A_{1}^{G}+\frac{i \varepsilon^{\mu P k N}}{M^{3}} A_{2}^{G}+\frac{i \varepsilon^{\mu P \Delta N}}{M^{3}} A_{3}^{G}+\frac{i \varepsilon^{\mu k \Delta N}}{M^{3}} A_{4}^{G}\right. \\
& +\frac{i \sigma^{\mu P} \gamma_{5}}{M} A_{5}^{G}+\frac{i \sigma^{\mu k} \gamma_{5}}{M} A_{6}^{G}+\frac{i \sigma^{\mu N} \gamma_{5}}{M} A_{7}^{G}+\frac{k^{\mu} i \sigma^{P N} \gamma_{5}}{M^{3}} A_{8}^{G}+\frac{\Delta^{\mu} i \sigma^{P N} \gamma_{5}}{M^{3}} A_{9}^{G} \\
& +\frac{N^{\mu} i \sigma^{P N} \gamma_{5}}{M^{3}} A_{10}^{G}+\frac{k^{\mu} i \sigma^{k N} \gamma_{5}}{M^{3}} A_{11}^{G}+\frac{\Delta^{\mu} i \sigma^{k N} \gamma_{5}}{M^{3}} A_{12}^{G}+\frac{N^{\mu} i \sigma^{k N} \gamma_{5}}{M^{3}} A_{13}^{G} \\
& \left.+\frac{P^{\mu} i \sigma^{\Delta N} \gamma_{5}}{M^{3}} A_{14}^{G}+\frac{\Delta^{\mu} i \sigma^{\Delta N} \gamma_{5}}{M^{3}} A_{15}^{G}+\frac{N^{\mu} i \sigma^{\Delta N} \gamma_{5}}{M^{3}} A_{16}^{G}\right] u(p, \lambda),
\end{aligned}
$$

where the GPCFs $A_{n}^{G}$ are scalar functions of $P, k, \Delta, N$, and $\eta$.

\section{A.5 Parameterization of the tensor Dirac bilinear}

A complete parameterization of the tensor Dirac bilinear in eq. (2.10) can be constructed from the parameterization of the pseudoscalar and the vector Dirac bilinears in eqs. (A.24) and (A.33). Respecting the antisymmetry of the tensor Dirac bilinear in eq. (2.10) it can be rewritten as

$$
\begin{aligned}
\bar{u}\left(p^{\prime}, \lambda^{\prime}\right) \Gamma_{\mathrm{T}}^{\mu \nu}(P, k, \Delta, N ; \eta) u(p, \lambda) \\
=\left(\delta_{\rho}^{\mu} \delta_{\sigma}^{\nu}-\delta_{\rho}^{\nu} \delta_{\sigma}^{\mu}\right) \bar{u}\left(p^{\prime}, \lambda^{\prime}\right)\left[\left(\frac{P^{\rho}}{M}-\frac{P^{2} N^{\rho}}{2 M^{3}}\right) \frac{M \Gamma_{\mathrm{T}}^{+\sigma}(P, k, \Delta, N ; \eta)}{P \cdot n}\right. \\
\quad+\frac{N^{\rho}}{M}\left(\delta_{\tau}^{\sigma}-\frac{P^{\sigma} N_{\tau}}{M^{2}}\right) \frac{(P \cdot n) \Gamma_{\mathrm{T}}^{-\tau}(P, k, \Delta, N ; \eta)}{M} \\
\left.\quad-\frac{i \varepsilon^{\rho \sigma P N}}{2 M^{2}} i \Gamma_{\mathrm{T}}^{12}(P, k, \Delta, N ; \eta)\right] u(p, \lambda),
\end{aligned}
$$

where from eqs. (A.1) - A.5) it follows that the structures $M \Gamma_{\mathrm{T}}^{+\mu} /(P \cdot n)$ and $(P \cdot n) \Gamma_{\mathrm{T}}^{-\mu} / M$ behave like vectors under parity whereas the structure $i \Gamma_{\mathrm{T}}^{12}$ behaves like a pseudoscalar. Therefore, inserting the respective parameterization from eqs. (A.24) and (A.33) into (A.43) 
a possible parameterization of the tensor Dirac bilinear in eq. (2.10) is given by

$$
\begin{aligned}
\bar{u}\left(p^{\prime}, \lambda^{\prime}\right) & \Gamma_{\mathrm{T}}^{\mu \nu}(P, k, \Delta, N ; \eta) u(p, \lambda) \\
= & \left(\delta_{\rho}^{\mu} \delta_{\sigma}^{\nu}-\delta_{\rho}^{\nu} \delta_{\sigma}^{\mu}\right) \bar{u}\left(p^{\prime}, \lambda^{\prime}\right)\left[( \frac { P ^ { \rho } } { M } - \frac { P ^ { 2 } N ^ { \rho } } { 2 M ^ { 3 } } ) \left(\frac{P^{\sigma}}{M} A_{1}+\frac{k^{\sigma}}{M} A_{2}+\frac{\Delta^{\sigma}}{M} A_{3}+\frac{N^{\sigma}}{M} A_{4}\right.\right. \\
& +\frac{i \sigma^{\sigma k}}{M} A_{5}+\frac{i \sigma^{\sigma \Delta}}{M} A_{6}+\frac{i \sigma^{\sigma N}}{M} A_{7}+\frac{P^{\sigma} i \sigma^{k \Delta}}{M^{3}} A_{8}+\frac{k^{\sigma} i \sigma^{k \Delta}}{M^{3}} A_{9}+\frac{N^{\sigma} i \sigma^{k \Delta}}{M^{3}} A_{10} \\
& +\frac{P^{\sigma} i \sigma^{k N}}{M^{3}} A_{11}+\frac{k^{\sigma} i \sigma^{k N}}{M^{3}} A_{12}+\frac{N^{\sigma} i \sigma^{k N}}{M^{3}} A_{13}+\frac{P^{\sigma} i \sigma^{\Delta N}}{M^{3}} A_{14}+\frac{\Delta^{\sigma} i \sigma^{\Delta N}}{M^{3}} A_{15} \\
& \left.+\frac{N^{\sigma} i \sigma^{\Delta N}}{M^{3}} A_{16}\right)+\frac{N^{\rho}}{M}\left(\delta_{\tau}^{\sigma}-\frac{P^{\sigma} N_{\tau}}{M^{2}}\right)\left(\frac{P^{\tau}}{M} A_{17}+\frac{k^{\tau}}{M} A_{18}+\frac{\Delta^{\tau}}{M} A_{19}+\frac{N^{\tau}}{M} A_{20}\right. \\
& +\frac{i \sigma^{\tau k}}{M_{21}}+\frac{i \sigma^{\tau \Delta}}{M} A_{22}+\frac{i \sigma^{\tau N}}{M} A_{23}+\frac{P^{\tau} i \sigma^{k \Delta}}{M^{3}} A_{24}+\frac{k^{\tau} i \sigma^{k \Delta}}{M^{3}} A_{25}+\frac{N^{\tau} i \sigma^{k \Delta}}{M^{3}} A_{26} \\
& +\frac{P^{\tau} i \sigma^{k N}}{M^{3}} A_{27}+\frac{k^{\tau} i \sigma^{k N}}{M^{3}} A_{28}+\frac{N^{\tau} i \sigma^{k N}}{M^{3}} A_{29}+\frac{P^{\tau} i \sigma^{\Delta N}}{M^{3}} A_{30}+\frac{\Delta^{\tau} i \sigma^{\Delta N}}{M^{3}} A_{31} \\
& \left.+\frac{N^{\tau} i \sigma^{\Delta N}}{M^{3}} A_{32}\right)-\frac{i \varepsilon^{\rho \sigma P N}}{2 M^{2}}\left(\frac{i \varepsilon^{P k \Delta N}}{M^{4}} A_{33}+\frac{i \sigma^{P N} \gamma_{5}}{M^{2}} A_{34}+\frac{i \sigma^{k N} \gamma_{5}}{M^{2}} A_{35}\right. \\
& \left.\left.+\frac{i \sigma^{\Delta N} \gamma_{5}}{M^{2}} A_{36}\right)\right] u(p, \lambda) .
\end{aligned}
$$

Of course, not all 36 structures in this parameterization are independent. It is therefore necessary to rearrange them in order to obtain a minimal set of structures for the parameterization of the tensor Dirac bilinear in eq. (2.10). By first performing all possible contractions in eq. (A.44) and after that using the $\sigma$-identity in (A.11) for the last three terms as well as the identity

$$
\epsilon^{\alpha \beta \gamma \delta} \epsilon^{\mu \nu \rho \sigma}=-\operatorname{det}\left(\begin{array}{cccc}
g^{\alpha \mu} & g^{\beta \mu} & g^{\gamma \mu} & g^{\delta \mu} \\
g^{\alpha \nu} & g^{\beta \nu} & g^{\gamma \nu} & g^{\delta \nu} \\
g^{\alpha \rho} & g^{\beta \rho} & g^{\gamma \rho} & g^{\delta \rho} \\
g^{\alpha \sigma} & g^{\beta \sigma} & g^{\gamma \sigma} & g^{\delta \sigma}
\end{array}\right)
$$

for all products of two $\epsilon$-tensors the number of independent structures already reduces significantly. Finally, the Gordon identity in eq. (A.7) allows one to express all tensor currents containing a $\sigma$-matrix contracted with $P$ in terms of scalar currents. This reduces the number of independent structures to 24 , which are given for example by

$$
\begin{aligned}
\bar{u}\left(p^{\prime}, \lambda^{\prime}\right) & \Gamma_{\mathrm{T}}^{\mu \nu}(P, k, \Delta, N ; \eta) u(p, \lambda) \\
= & \left(\delta_{\rho}^{\mu} \delta_{\sigma}^{\nu}-\delta_{\rho}^{\nu} \delta_{\sigma}^{\mu}\right) \bar{u}\left(p^{\prime}, \lambda^{\prime}\right)\left[\frac{P^{\rho} k^{\sigma}}{M^{2}} A_{1}^{H}+\frac{P^{\rho} \Delta^{\sigma}}{M^{2}} A_{2}^{H}+\frac{P^{\rho} N^{\sigma}}{M^{2}} A_{3}^{H}+\frac{k^{\rho} \Delta^{\sigma}}{M^{2}} A_{4}^{H}\right. \\
& +\frac{k^{\rho} N^{\sigma}}{M^{2}} A_{5}^{H}+\frac{\Delta^{\rho} N^{\sigma}}{M^{2}} A_{6}^{H}+i \sigma^{\rho \sigma} A_{7}^{H}+\frac{P^{\rho} i \sigma^{\sigma k}}{M^{2}} A_{8}^{H}+\frac{N^{\rho} i \sigma^{\sigma k}}{M^{2}} A_{9}^{H} \\
& +\frac{P^{\rho} i \sigma^{\sigma \Delta}}{M^{2}} A_{10}^{H}+\frac{N^{\rho} i \sigma^{\sigma \Delta}}{M^{2}} A_{11}^{H}+\frac{P^{\rho} i \sigma^{\sigma N}}{M^{2}} A_{12}^{H}+\frac{k^{\rho} i \sigma^{\sigma N}}{M^{2}} A_{13}^{H}+\frac{\Delta^{\rho} i \sigma^{\sigma N}}{M^{2}} A_{14}^{H} \\
& +\frac{N^{\rho} i \sigma^{\sigma N}}{M^{2}} A_{15}^{H}+\frac{P^{\rho} k^{\sigma} i \sigma^{k \Delta}}{M^{4}} A_{16}^{H}+\frac{P^{\rho} N^{\sigma} i \sigma^{k \Delta}}{M^{4}} A_{17}^{H}+\frac{k^{\rho} N^{\sigma} i \sigma^{k \Delta}}{M^{4}} A_{18}^{H}
\end{aligned}
$$




$$
\begin{aligned}
& +\frac{P^{\rho} k^{\sigma} i \sigma^{k N}}{M^{4}} A_{19}^{H}+\frac{P^{\rho} N^{\sigma} i \sigma^{k N}}{M^{4}} A_{20}^{H}+\frac{k^{\rho} N^{\sigma} i \sigma^{k N}}{M^{4}} A_{21}^{H}+\frac{P^{\rho} \Delta^{\sigma} i \sigma^{\Delta N}}{M^{4}} A_{22}^{H} \\
& \left.+\frac{P^{\rho} N^{\sigma} i \sigma^{\Delta N}}{M^{4}} A_{23}^{H}+\frac{\Delta^{\rho} N^{\sigma} i \sigma^{\Delta N}}{M^{4}} A_{24}^{H}\right] u(p, \lambda),
\end{aligned}
$$

where the GPCFs $A_{n}^{H}$ are scalar functions of $P, k, \Delta, N$, and $\eta$ and are related to the structures $A_{n}$ in eq. (A.44) by

$$
\begin{aligned}
& A_{1}^{H}=A_{2}+\frac{\Delta \cdot N}{M^{2}} A_{33}, \\
& A_{2}^{H}=A_{3}-\frac{k \cdot N}{M^{2}} A_{33}, \\
& A_{3}^{H}=\frac{P^{2}}{2 M^{2}} A_{1}+A_{4}+\frac{k \cdot N}{M^{2}} A_{18}+\frac{\Delta \cdot N}{M^{2}} A_{19} \\
& -\frac{(P \cdot k)(\Delta \cdot N)}{M^{4}} A_{33}-\frac{\Delta \cdot N}{2 M^{2}} A_{34}, \\
& A_{4}^{H}=A_{33}, \\
& A_{5}^{H}=\frac{P^{2}}{2 M^{2}} A_{2}-A_{18}+\frac{P^{2}(\Delta \cdot N)}{M^{4}} A_{33}-\frac{\Delta \cdot N}{2 M^{2}} A_{35}, \\
& A_{6}^{H}=\frac{P^{2}}{2 M^{2}} A_{3}-A_{19}-\frac{P^{2}(k \cdot N)-(P \cdot k)(P \cdot N)}{M^{4}} A_{33} \\
& +\frac{1}{2} A_{34}+\frac{k \cdot N}{2 M^{2}} A_{35}, \\
& A_{7}^{H}=\frac{1}{2} A_{34}+\frac{k \cdot N}{2 M^{2}} A_{35}+\frac{\Delta \cdot N}{2 M^{2}} A_{36}, \\
& A_{8}^{H}=A_{5} \text {, } \\
& A_{9}^{H}=-\frac{P^{2}}{2 M^{2}} A_{5}+A_{21}, \\
& A_{10}^{H}=A_{6}, \\
& A_{11}^{H}=-\frac{P^{2}}{2 M^{2}} A_{6}+A_{22}, \\
& A_{12}^{H}=A_{7}+A_{34} \text {, } \\
& A_{13}^{H}=A_{35} \text {, } \\
& A_{14}^{H}=A_{36} \text {, } \\
& A_{15}^{H}=-\frac{P^{2}}{2 M^{2}} A_{7}+A_{23}-\frac{P^{2}}{M^{2}} A_{34}-\frac{P \cdot k}{M^{2}} A_{35}, \\
& A_{16}^{H}=A_{9}, \\
& A_{17}^{H}=\frac{P^{2}}{2 M^{2}} A_{8}+A_{10}+\frac{k \cdot N}{M^{2}} A_{25}, \\
& A_{18}^{H}=\frac{P^{2}}{2 M^{2}} A_{9}-A_{25}, \\
& A_{19}^{H}=A_{12} \text {, } \\
& A_{20}^{H}=\frac{P^{2}}{2 M^{2}} A_{11}+A_{13}-A_{21}+\frac{k \cdot N}{M^{2}} A_{28}, \\
& A_{21}^{H}=\frac{P^{2}}{2 M^{2}} A_{12}-A_{28},
\end{aligned}
$$




$$
\begin{aligned}
& A_{22}^{H}=A_{15}, \\
& A_{23}^{H}=\frac{P^{2}}{2 M^{2}} A_{14}+A_{16}-A_{22}+\frac{\Delta \cdot N}{M^{2}} A_{31}, \\
& A_{24}^{H}=\frac{P^{2}}{2 M^{2}} A_{15}-A_{31} .
\end{aligned}
$$

\section{B. Relations between GTMDs and GPCFs}

Here the explicit relations between the leading twist GTMDs in eqs. (3.6)-(3.8) and the

GPCFs in eqs. (2.19)-(2.21) are listed. For brevity we leave out the arguments of the functions. Straightforward calculation leads to the results

$$
\begin{aligned}
& F_{1,1}=2 P^{+} \int d k^{-}\left[A_{1}^{F}+x A_{2}^{F}-2 \xi A_{3}^{F}+\xi\left(x A_{5}^{F}-2 \xi A_{6}^{F}\right)\right. \\
& +\frac{2 \xi k \cdot \Delta+x \Delta^{2}}{2 M^{2}}\left(A_{8}^{F}+x A_{9}^{F}\right) \\
& \left.-x \xi\left(A_{11}^{F}+x A_{12}^{F}\right)+2 \xi^{2}\left(A_{14}^{F}-2 \xi A_{15}^{F}\right)\right], \\
& F_{1,2}=2 P^{+} \int d k^{-}\left[A_{5}^{F}-\frac{2 \xi P^{2}}{M^{2}}\left(A_{8}^{F}+x A_{9}^{F}\right)-\left(A_{11}^{F}+x A_{12}^{F}\right)\right], \\
& F_{1,3}=2 P^{+} \int d k^{-}\left[A_{6}^{F}-\frac{x P^{2}-P \cdot k}{M^{2}}\left(A_{8}^{F}+x A_{9}^{F}\right)-\left(A_{14}^{F}-2 \xi A_{15}^{F}\right)\right] \text {, } \\
& F_{1,4}=2 P^{+} \int d k^{-}\left[A_{8}^{F}+x A_{9}^{F}\right], \\
& G_{1,1}=2 P^{+} \int d k^{-}\left[A_{1}^{G}\right] \\
& G_{1,2}=2 P^{+} \int d k^{-}\left[A_{6}^{G}-\left(x A_{11}^{G}-2 \xi A_{12}^{G}\right)\right], \\
& G_{1,3}=2 P^{+} \int d k^{-}\left[-\left(A_{14}^{G}-2 \xi A_{15}^{G}\right)\right], \\
& G_{1,4}=2 P^{+} \int d k^{-}\left[A_{5}^{G}+x A_{6}^{G}-x A_{8}^{G}+2 \xi A_{9}^{G}-x\left(x A_{11}^{G}-2 \xi A_{12}^{G}\right)\right. \\
& \left.+2 \xi\left(A_{14}^{G}-2 \xi A_{15}^{G}\right)\right], \\
& H_{1,1}=2 P^{+} \int d k^{-}\left[A_{1}^{H}+2 \xi A_{4}^{H}+\frac{2 \xi k \cdot \Delta+x \Delta^{2}}{2 M^{2}} A_{16}^{H}-x \xi A_{19}^{H}\right], \\
& H_{1,2}=2 P^{+} \int d k^{-}\left[A_{2}^{H}+x A_{4}^{H}-\frac{1}{2} x A_{8}^{H}+\xi A_{10}^{H}+2 \xi^{2} A_{22}^{H}\right], \\
& H_{1,3}=2 P^{+} \int d k^{-}\left[2 A_{7}^{H}+\frac{x P^{2}-P \cdot k}{M^{2}} A_{8}^{H}-\frac{2 \xi P^{2}}{M^{2}} A_{10}^{H}-\left(A_{12}^{H}+x A_{13}^{H}-2 \xi A_{14}^{H}\right)\right. \\
& -\frac{P^{2}\left(2 \xi k^{2}+x k \cdot \Delta\right)-P \cdot k(2 \xi P \cdot k+k \cdot \Delta)}{M^{4}} A_{16}^{H} \\
& \left.-\frac{x^{2} P^{2}-2 x P \cdot k+k^{2}}{M^{2}} A_{19}^{H}-\frac{4 \xi^{2} P^{2}+\Delta^{2}}{M^{2}} A_{22}^{H}\right],
\end{aligned}
$$




$$
\begin{aligned}
& H_{1,4}=2 P^{+} \int d k^{-}\left[-A_{19}^{H}-\frac{2 \xi P^{2}}{M^{2}} A_{16}^{H}\right], \\
& H_{1,5}=2 P^{+} \int d k^{-}\left[-\frac{x P^{2}-P \cdot k}{M^{2}} A_{16}^{H}\right], \\
& H_{1,6}=2 P^{+} \int d k^{-}\left[-A_{22}^{H}\right], \\
& H_{1,7}=2 P^{+} \int d k^{-}\left[-A_{8}^{H}-\frac{2 x \xi P^{2}-2 \xi P \cdot k-k \cdot \Delta}{M^{2}} A_{16}^{H}\right], \\
& H_{1,8}=2 P^{+} \int d k^{-}\left[-A_{10}^{H}-\frac{x^{2} P^{2}-2 x P \cdot k+k^{2}}{M^{2}} A_{16}^{H}\right] .
\end{aligned}
$$

\section{Model calculation of GTMDs}

For illustrative purposes and in order to get a first estimate we calculate all the leading twist GTMDs in the scalar spectator diquark model of the nucleon (see, e.g., ref. [58]) by restricting ourselves to lowest nontrivial order in perturbation theory. The Lagrangian of the diquark model reads

$$
\begin{aligned}
\mathcal{L}_{\mathrm{SDM}}(x)= & \bar{\Psi}(x)\left(i \gamma^{\mu} \partial_{\mu}-M\right) \Psi(x)+\bar{\psi}(x)\left(i \gamma^{\mu} \partial_{\mu}-m_{q}\right) \psi(x) \\
& +\partial^{\mu} \varphi^{*}(x) \partial_{\mu} \varphi(x)-m_{s}^{2} \varphi^{*}(x) \varphi(x) \\
& +g\left[\bar{\psi}(x) \Psi(x) \varphi^{*}(x)+\bar{\Psi}(x) \psi(x) \varphi(x)\right],
\end{aligned}
$$

where $\Psi$ denotes the nucleon field, $\psi$ the quark field, and $\varphi$ the scalar diquark field. The essential ingredient of the model is a three-point interaction between the target, quarks, and diquarks, with the coupling constant $g$. This framework allows one to carry out perturbative calculations. All the results for parton distributions given below contain the coupling $g$ to the second power. Notice also that the condition $M<m_{q}+m_{s}$ has to be fulfilled in order to have a stable target state.

The lowest order contribution to the generalized $k_{T}$-dependent correlator in eq. (3.1) comes from the tree-level diagrams depicted in figure 3. These diagrams can be evaluated in a straightforward manner yielding

$$
\begin{aligned}
& W_{\lambda \lambda^{\prime}}^{[\Gamma]}\left(P, x, \vec{k}_{T}, \Delta, N ; \eta\right) \\
& =\frac{g^{2}(1-x)\left(x^{2}-\xi^{2}\right)}{4(2 \pi)^{3} P^{+}}\left[\frac{N\left(k_{1}^{-}\right)}{D_{+} D_{-}} \Theta(1-x)-\frac{N\left(k_{2}^{-}\right)}{D_{+}\left(D_{-}-D_{+}\right)} \Theta(\xi-x)\right. \\
& \left.\quad-\frac{N\left(k_{3}^{-}\right)}{\left(D_{+}-D_{-}\right) D_{-}} \Theta(-\xi-x)\right] \Theta(1-|\xi|),
\end{aligned}
$$

where the numerator $N\left(k^{-}\right)$is given by

$$
N\left(k^{-}\right)=\bar{u}\left(p^{\prime}, \lambda^{\prime}\right)\left(\not k+\frac{1}{2} \not \Delta+m_{q}\right) \Gamma\left(\not k-\frac{1}{2} \not \Delta+m_{q}\right) u(p, \lambda),
$$




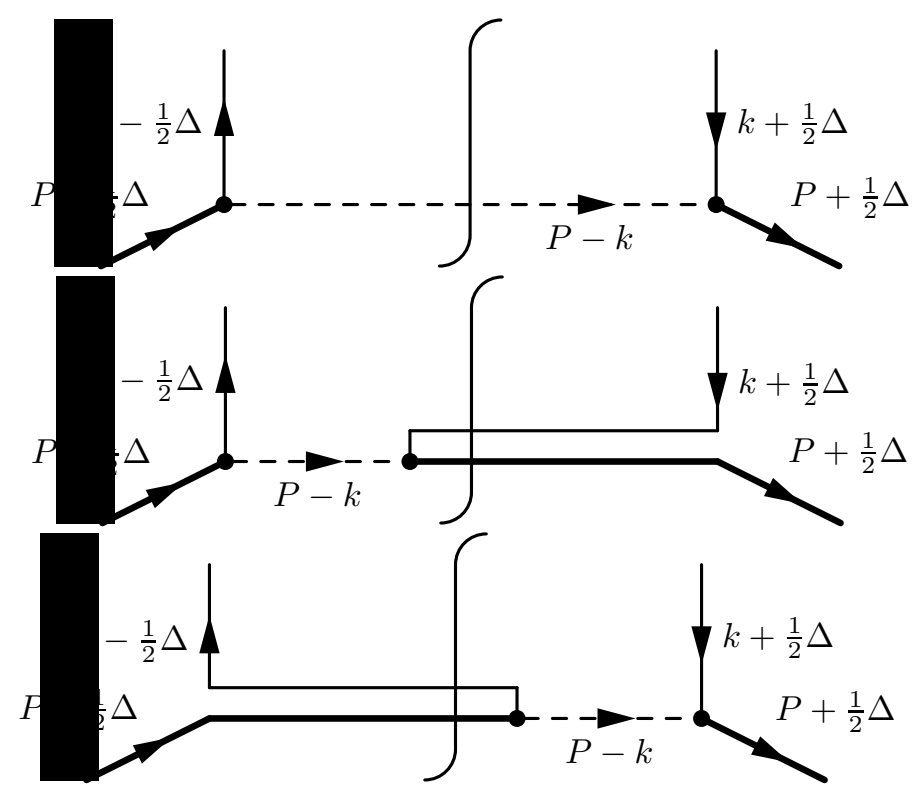

Figure 3: Lowest nontrivial order diagrams contributing to the GTMDs in the scalar spectator diquark model. The second and third diagram are only relevant for the ERBL region which is characterized by $|x| \leq|\xi|$.

the denominators $D_{ \pm}$by

$$
\begin{aligned}
D_{ \pm}= & \frac{x \pm \xi}{1 \mp \xi}\left((1 \mp \xi) \vec{k}_{T} \pm \frac{1}{2}(1-x) \vec{\Delta}_{T}\right)^{2}+\left(x^{2}-\xi^{2}\right) m_{s}^{2} \\
& +(1-x)(x \pm \xi) m_{q}^{2}-\frac{(1-x)\left(x^{2}-\xi^{2}\right)}{1 \mp \xi} M^{2}
\end{aligned}
$$

and $k^{-}$is fixed by the cuts in the diagrams to be

$$
\begin{aligned}
& k_{1}^{-}=\frac{\frac{1}{4} \vec{\Delta}_{T}^{2}+M^{2}}{2\left(1-\xi^{2}\right) P^{+}}-\frac{\vec{k}_{T}^{2}+m_{s}^{2}}{2(1-x) P^{+}}, \\
& k_{2}^{-}=-\xi \frac{\frac{1}{4} \vec{\Delta}_{T}^{2}+M^{2}}{2\left(1-\xi^{2}\right) P^{+}}+\frac{\left(\vec{k}_{T}+\frac{1}{2} \vec{\Delta}_{T}\right)^{2}+m_{q}^{2}}{2(x-\xi) P^{+}} \\
& k_{3}^{-}=\xi \frac{\frac{1}{4} \vec{\Delta}_{T}^{2}+M^{2}}{2\left(1-\xi^{2}\right) P^{+}}+\frac{\left(\vec{k}_{T}-\frac{1}{2} \vec{\Delta}_{T}\right)^{2}+m_{q}^{2}}{2(x+\xi) P^{+}} .
\end{aligned}
$$

Since the calculation is carried out only to lowest order in perturbation theory, no effect due to the Wilson line enters. As a consequence, the correlator (C.2) actually does not depend on the parameter $\eta$.

Using now the expression (C.2) and the definitions for the GTMDs in eqs. (3.6)- 3.8) it is possible to calculate the leading twist GTMDs. As for leading twist the numerator $N\left(k^{-}\right)$in eq. C.3 does actually not depend on $k^{-}$, the final expressions are not too 
complicated. They read ${ }^{4}$

$$
\begin{aligned}
& F_{1,1}^{e}=C\left[\left(1-\xi^{2}\right)\left(\vec{k}_{T}^{2}+\left(m_{q}+M\right)^{2}\right)+(1-x)\left(\xi \vec{k}_{T} \cdot \vec{\Delta}_{T}-2\left(m_{q}+x M\right) M\right)\right. \\
& \left.-\frac{1}{4}(1-x)^{2}\left(\vec{\Delta}_{T}^{2}+4 M^{2}\right)\right] \\
& F_{1,2}^{e}=2 C\left[\frac{\xi(1-x)}{4\left(1-\xi^{2}\right)}\left(\vec{\Delta}_{T}^{2}+4 M^{2}\right)\right] \text {, } \\
& F_{1,3}^{e}=\frac{1}{2} C\left[\left(\vec{k}_{T}^{2}+\left(m_{q}+M\right)^{2}\right)-\frac{(1-x)^{2}}{4\left(1-\xi^{2}\right)}\left(\vec{\Delta}_{T}^{2}+4 M^{2}\right)\right], \\
& F_{1,4}^{e}=-C\left[(1-x) M^{2}\right] \text {, } \\
& G_{1,1}^{e}=-C\left[(1-x) M^{2}\right] \text {, } \\
& G_{1,2}^{e}=-2 C\left[\frac{1-x}{4\left(1-\xi^{2}\right)}\left(\vec{\Delta}_{T}^{2}+4 M^{2}\right)-\left(m_{q}+M\right) M\right] \text {, } \\
& G_{1,3}^{e}=-\frac{1}{2} C\left[\xi\left(\vec{k}_{T}^{2}-\left(m_{q}+M\right)^{2}\right)-(1-x) \vec{k}_{T} \cdot \vec{\Delta}_{T}\right. \\
& \left.-\frac{\xi(1-x)^{2}}{4\left(1-\xi^{2}\right)}\left(\vec{\Delta}_{T}^{2}+4 M^{2}\right)\right] \text {, } \\
& G_{1,4}^{e}=-C\left[\left(1-\xi^{2}\right)\left(\vec{k}_{T}^{2}-\left(m_{q}+M\right)^{2}\right)+(1-x)\left(\xi \vec{k}_{T} \cdot \vec{\Delta}_{T}+2\left(m_{q}+M\right) M\right)\right. \\
& \left.-\frac{1}{4}(1-x)^{2}\left(\vec{\Delta}_{T}^{2}+4 M^{2}\right)\right] \\
& H_{1,1}^{e}=2 C\left[\xi(1-x) M^{2}\right] \\
& H_{1,2}^{e}=C\left[(1-x)\left(m_{q}+x M\right) M\right] \text {, } \\
& H_{1,3}^{e}=C\left[\left(\vec{k}_{T}^{2}+\left(m_{q}+M\right)^{2}\right)+\frac{\xi\left(m_{q}+M\right)}{M} \frac{\vec{k}_{T}^{2} \vec{\Delta}_{T}^{2}-\left(\vec{k}_{T} \cdot \vec{\Delta}_{T}\right)^{2}}{\vec{k}_{T} \cdot \vec{\Delta}_{T}}\right. \\
& \left.-\frac{(1-x)\left(\left(m_{q}+M\right)+\left(m_{q}+x M\right)\right)}{4\left(1-\xi^{2}\right) M}\left(\vec{\Delta}_{T}^{2}+4 M^{2}\right)\right], \\
& H_{1,4}^{e}=-C\left[\frac{\xi \vec{\Delta}_{T}^{2}}{\vec{k}_{T} \cdot \vec{\Delta}_{T}}\left(m_{q}+M\right) M+2 M^{2}\right] \text {, } \\
& H_{1,5}^{e}=C\left[\xi\left(m_{q}+M\right) M\right] \text {, } \\
& H_{1,6}^{e}=-C\left[\left(\frac{\xi \vec{k}_{T}^{2}}{\vec{k}_{T} \cdot \vec{\Delta}_{T}}-\frac{1}{2}(1-x)\right)\left(m_{q}+M\right) M\right], \\
& H_{1,7}^{e}=-2 C\left[\left(1-\xi^{2}\right)\left(m_{q}+M\right) M-(1-x) M^{2}\right],
\end{aligned}
$$

\footnotetext{
${ }^{4}$ In ref. [1] we obtained results for the GTMDs of a spin-0 target in a simple spectator model. Those results are only complete for $|\xi| \leq x \leq 1$. But note also that this specific kinematical region includes the case $\xi=0$ which is relevant for discussing potential nontrivial relations between GPDs and TMDs.
} 


$$
H_{1,8}^{e}=-C\left[\xi(1-x)\left(m_{q}+M\right) M\right]
$$

with $^{5}$

$$
C=\left\{\begin{array}{cl}
0 & \text { for } x \geq 1 \text { (unphysical region), } \\
\frac{g^{2}(1-x)\left(x^{2}-\xi^{2}\right)}{2(2 \pi)^{3} D_{+} D_{-}} & \text {for }|\xi| \leq x \leq 1 \text { (DGLAP region for quarks), } \\
\frac{g^{2}(1-x)\left(x^{2}-\xi^{2}\right)}{2(2 \pi)^{3}\left(D_{+}-D_{-}\right) D_{-}} & \text {for }-1 \leq-\xi \leq x \leq \xi \leq 1 \text { (ERBL region for } \xi \geq 0 \text { ), } \\
\frac{g^{2}(1-x)\left(x^{2}-\xi^{2}\right)}{2(2 \pi)^{3} D_{+}\left(D_{-}-D_{+}\right)} & \text {for }-1 \leq \xi \leq x \leq-\xi \leq 1 \text { (ERBL region for } \xi \leq 0 \text { ), } \\
0 & \text { for }-1 \leq x \leq-|\xi| \text { (DGLAP region for antiquarks), } \\
0 & \text { for } x \leq-1 \text { (unphysical region). }
\end{array}\right.
$$

To shorten the notation we have suppressed the arguments of the GTMDs. All (naïve) T-odd parts of GTMDs vanish to lowest order in perturbation theory investigated here. To get nonzero results for these functions requires considering at least one-loop corrections that include effects from the Wilson line. We checked that the limits of TMDs and GPDs for $\xi=0$ agree with the results found in [24].

\section{References}

[1] S. Meissner, A. Metz, M. Schlegel, and K. Goeke, Generalized parton correlation functions for a spin-0 hadron, JHEP 08 (2008) 038, arXiv:0805.3165.

[2] K. Goeke, A. Metz, and M. Schlegel, Parameterization of the quark-quark correlator of a spin-1/2 hadron, Phys. Lett. B618 (2005) 90-96, hep-ph/0504130.

[3] J. C. Collins, T. C. Rogers, and A. M. Stasto, Fully unintegrated parton correlation functions and factorization in lowest order hard scattering, Phys. Rev. D77 (2008) 085009, arXiv:0708.2833 [hep-ph]].

[4] T. C. Rogers, Next-to-Leading Order Hard Scattering Using Fully Unintegrated Parton Distribution Functions, Phys. Rev. D78 (2008) 074018, arXiv:0807.2430.

[5] D. Müller, D. Robaschik, B. Geyer, F. M. Dittes, and J. Horejsi, Wave functions, evolution equations and evolution kernels from light-ray operators of QCD, Fortschr. Phys. 42 (1994) 101, hep-ph/9812448.

[6] X.-D. Ji, Gauge invariant decomposition of nucleon spin, Phys. Rev. Lett. 78 (1997) 610-613, hep-ph/9603249.

[7] A. V. Radyushkin, Scaling Limit of Deeply Virtual Compton Scattering, Phys. Lett. B380 (1996) 417-425, hep-ph/9604317.

\footnotetext{
${ }^{5}$ Note that in the context of GPDs one typically only considers the case $\xi \geq 0$ as this condition is satisfied for all known processes where GPDs can be measured. Here we do not use this restriction and take negative values of $\xi$ into account as well.
} 
[8] K. Goeke, M. V. Polyakov, and M. Vanderhaeghen, Hard exclusive reactions and the structure of hadrons, Prog. Part. Nucl. Phys. 47 (2001) 401-515, hep-ph/0106012.

[9] M. Diehl, Generalized parton distributions, Phys. Rept. 388 (2003) 41-277, hep-ph/0307382.

[10] A. V. Belitsky and A. V. Radyushkin, Unraveling hadron structure with generalized parton distributions, Phys. Rept. 418 (2005) 1-387, hep-ph/0504030.

[11] S. Boffi and B. Pasquini, Generalized parton distributions and the structure of the nucleon, Riv. Nuovo Cim. 30 (2007) 387, arXiv:0711.2625.

[12] P. J. Mulders and R. D. Tangerman, The complete tree-level result up to order $1 / Q$ for polarized deep-inelastic leptoproduction, Nucl. Phys. B461 (1996) 197-237, hep-ph/9510301.

[13] V. Barone, A. Drago, and P. G. Ratcliffe, Transverse polarisation of quarks in hadrons, Phys. Rept. 359 (2002) 1-168, hep-ph/0104283.

[14] A. Bacchetta, M. Diehl, K. Goeke, A. Metz, P. J. Mulders, and M. Schlegel, Semi-inclusive deep inelastic scattering at small transverse momentum, JHEP 02 (2007) 093, hep-ph/0611265.

[15] U. D'Alesio and F. Murgia, Azimuthal and Single Spin Asymmetries in Hard Scattering Processes, Prog. Part. Nucl. Phys. 61 (2008) 394-454, arXiv:0712.4328.

[16] X.-d. Ji, Viewing the proton through 'color'-filters, Phys. Rev. Lett. 91 (2003) 062001, hep-ph/0304037.

[17] A. V. Belitsky, X.-d. Ji, and F. Yuan, Quark imaging in the proton via quantum phase-space distributions, Phys. Rev. D69 (2004) 074014, hep-ph/0307383.

[18] M. Burkardt, Impact parameter dependent parton distributions and transverse single spin asymmetries, Phys. Rev. D66 (2002) 114005, hep-ph/0209179.

[19] M. Burkardt, Chromodynamic lensing and transverse single spin asymmetries, Nucl. Phys. A735 (2004) 185-199, hep-ph/0302144.

[20] M. Burkardt and D. S. Hwang, Sivers asymmetry and generalized parton distributions in impact parameter space, Phys. Rev. D69 (2004) 074032, hep-ph/0309072.

[21] M. Diehl and P. Hägler, Spin densities in the transverse plane and generalized transversity distributions, Eur. Phys. J. C44 (2005) 87-101, hep-ph/0504175.

[22] M. Burkardt, Transverse deformation of parton distributions and transversity decomposition of angular momentum, Phys. Rev. D72 (2005) 094020, hep-ph/0505189.

[23] Z. Lu and I. Schmidt, Connection between the Sivers function and the anomalous magnetic moment, Phys. Rev. D75 (2007) 073008, hep-ph/0611158.

[24] S. Meissner, A. Metz, and K. Goeke, Relations between generalized and transverse momentum dependent parton distributions, Phys. Rev. D76 (2007) 034002, hep-ph/0703176.

[25] B. Pasquini, S. Cazzaniga, and S. Boffi, Transverse momentum dependent parton distributions in a light-cone quark model, Phys. Rev. D78 (2008) 034025, arXiv:0806.2298.

[26] D. W. Sivers, Single spin production asymmetries from the hard scattering of point-like constituents, Phys. Rev. D41 (1990) 83. 
[27] D. W. Sivers, Hard scattering scaling laws for single spin production asymmetries, Phys. Rev. D43 (1991) 261-263.

[28] HERMES Collaboration, A. Airapetian et al., Single-spin asymmetries in semi-inclusive deep-inelastic scattering on a transversely polarized hydrogen target, Phys. Rev. Lett. 94 (2005) 012002, hep-ex/0408013.

[29] COMPASS Collaboration, V. Y. Alexakhin et al., First measurement of the transverse spin asymmetries of the deuteron in semi-inclusive deep inelastic scattering, Phys. Rev. Lett. 94 (2005) 202002, hep-ex/0503002.

[30] COMPASS Collaboration, E. S. Ageev et al., A new measurement of the Collins and Sivers asymmetries on a transversely polarised deuteron target, Nucl. Phys. B765 (2007) 31-70, hep-ex/0610068.

[31] HERMES Collaboration, A. Airapetian et al., Observation of the Naive-T-odd Sivers Effect in Deep- Inelastic Scattering, arXiv:0906.3918.

[32] S. Meissner, A. Metz, and M. Schlegel, Generalized transverse momentum dependent parton distributions of the nucleon, arXiv:0807.1154.

[33] M. Vanderhaeghen, P. A. M. Guichon, and M. Guidal, Deeply virtual electroproduction of photons and mesons on the nucleon: Leading order amplitudes and power corrections, Phys. Rev. D60 (1999) 094017, hep-ph/9905372.

[34] M. Diehl and W. Kugler, Next-to-leading order corrections in exclusive meson production, Eur. Phys. J. C52 (2007) 933-966, arXiv:0708.1121 [hep-ph]].

[35] S. V. Goloskokov and P. Kroll, The role of the quark and gluon GPDs in hard vector-meson electroproduction, Eur. Phys. J. C53 (2008) 367-384, arXiv:0708.3569 [hep-ph].

[36] A. D. Martin, M. G. Ryskin, and T. Teubner, $Q^{2}$-dependence of diffractive vector meson electroproduction, Phys. Rev. D62 (2000) 014022, hep-ph/9912551.

[37] V. A. Khoze, A. D. Martin, and M. G. Ryskin, Can the Higgs be seen in rapidity gap events at the Tevatron or the LHC?, Eur. Phys. J. C14 (2000) 525-534, hep-ph/0002072.

[38] FP420 R and D Collaboration, M. G. Albrow et al., The FP420 RESD Project: Higgs and New Physics with forward protons at the $L H C$, arXiv:0806.0302.

[39] A. D. Martin, M. G. Ryskin, and V. A. Khoze, Forward Physics at the LHC, arXiv:0903.2980.

[40] A. D. Martin and M. G. Ryskin, Unintegrated generalised parton distributions, Phys. Rev. D64 (2001) 094017, hep-ph/0107149.

[41] K. Goeke, A. Metz, P. V. Pobylitsa, and M. V. Polyakov, Lorentz invariance relations among parton distributions revisited, Phys. Lett. B567 (2003) 27-30, hep-ph/0302028.

[42] J. C. Collins, Leading-twist single-transverse-spin asymmetries: Drell-Yan and deep-inelastic scattering, Phys. Lett. B536 (2002) 43-48, hep-ph/0204004].

[43] J. C. Collins and D. E. Soper, Parton distribution and decay functions, Nucl. Phys. B194 (1982) 445.

[44] J. C. Collins and F. Hautmann, Infrared divergences and non-lightlike eikonal lines in Sudakov processes, Phys. Lett. B472 (2000) 129-134, hep-ph/9908467. 
[45] J. C. Collins and F. Hautmann, Soft gluons and gauge-invariant subtractions in NLO parton-shower Monte Carlo event generators, JHEP 03 (2001) 016, hep-ph/0009286.

[46] X.-d. Ji and F. Yuan, Parton distributions in light-cone gauge: Where are the final-state interactions?, Phys. Lett. B543 (2002) 66-72, hep-ph/0206057.

[47] A. V. Belitsky, X.-d. Ji, and F. Yuan, Final state interactions and gauge invariant parton distributions, Nucl. Phys. B656 (2003) 165-198, hep-ph/0208038.

[48] J. C. Collins and A. Metz, Universality of soft and collinear factors in hard-scattering factorization, Phys. Rev. Lett. 93 (2004) 252001, hep-ph/0408249.

[49] I. O. Cherednikov and N. G. Stefanis, Renormalization, Wilson lines, and transverse-momentum dependent parton distribution functions, Phys. Rev. D77 (2008) 094001, arXiv:0710.1955 [hep-ph].

[50] I. O. Cherednikov and N. G. Stefanis, Wilson lines and transverse-momentum dependent parton distribution functions: A renormalization-group analysis, Nucl. Phys. B802 (2008) 146-179, arXiv:0802.2821.

[51] J. C. Collins, What exactly is a parton density?, Acta Phys. Polon. B34 (2003) 3103, hep-ph/0304122.

[52] F. Hautmann, Endpoint singularities in unintegrated parton distributions, Phys. Lett. B655 (2007) 26-31, hep-ph/0702196.

[53] J. Collins, Rapidity divergences and valid definitions of parton densities, PoS LC2008 (2008) 028, arXiv:0808.2665.

[54] M. Diehl, Generalized parton distributions with helicity flip, Eur. Phys. J. C19 (2001) 485-492, hep-ph/0101335.

[55] D. V. Kiptily and M. V. Polyakov, Genuine twist-3 contributions to the generalized parton distributions from instantons, Eur. Phys. J. C37 (2004) 105-114, hep-ph/0212372.

[56] D. Boer and P. J. Mulders, Time-reversal odd distribution functions in leptoproduction, Phys. Rev. D57 (1998) 5780-5786, hep-ph/9711485.

[57] B. Pasquini, private communication, 2009.

[58] S. J. Brodsky, D. S. Hwang, and I. Schmidt, Final-state interactions and single-spin asymmetries in semi-inclusive deep inelastic scattering, Phys. Lett. B530 (2002) 99-107, hep-ph/0201296. 